\title{
1 Common Marmoset Gut Microbiome Profiles in Health and Intestinal Disease
}

2 Alexander Sheh ${ }^{1 \dagger}$,*, Stephen C. Artim ${ }^{1 \dagger}$, Monika A. Burns ${ }^{1}$, Jose Arturo Molina-Mora ${ }^{2}$, Mary Anne Lee $^{1,3}$, JoAnn Dzink-Fox ${ }^{1}$, Sureshkumar Muthupalani ${ }^{1}$, James G. Fox ${ }^{1, *}$

${ }^{2}$ Centro de Investigación en Enfermedades Tropicales (CIET), Universidad de Costa Rica, San José, Costa Rica

${ }^{3}$ Department of Biological Sciences, Wellesley College, Wellesley, Massachusetts

*Corresponding author(s).Email: alexsheh@mit.edu and jgfox@mit.edu

These authors contributed equally to this work

\section{Abstract}

Chronic gastrointestinal (GI) diseases are the most common diseases in captive marmosets. The

11 gut microbiome of healthy $(\mathrm{n}=91)$, inflammatory bowel disease (IBD) $(\mathrm{n}=59)$, and duodenal ulcer/stricture $(n=23)$ captive marmosets was characterized. Healthy marmosets exhibited a "humanized," Bacteroidetes-dominant microbiome. Despite standardized conditions, cohorts subdivided into Prevotella- and Bacteroides-dominant groups based on marmoset source. IBD was highest in a Prevotella-dominant cohort while strictures were highest in a Bacteroides-dominant cohort. Stricture-associated dysbiosis was characterized by Anaerobiospirillum loss and

17 Clostridium perfringens increases. Stricture tissue presented upregulation of lipid metabolism genes and increased abundance of $C$. perfringens, a causative agent of GI diseases and intestinal strictures in humans. IBD was associated with a lower Bacteroides:P. copri ratio within each source. Consistent with Prevotella-linked diseases, pro-inflammatory genes were upregulated.

21 This report highlights the humanization of the captive marmoset microbiome and its potential as a 
bioRxiv preprint doi: https://doi.org/10.1101/2020.08.27.268524; this version posted August 27, 2020. The copyright holder for this preprint (which was not certified by peer review) is the author/funder, who has granted bioRxiv a license to display the preprint in perpetuity. It is made available under aCC-BY-NC-ND 4.0 International license.

22 "humanized" animal model of $C$. perfringens-induced enteritis/strictures and $P$. copri-associated

23 IBD.

24 Keywords: marmoset, Prevotella copri, Clostridium perfringens, inflammatory bowel disease,

25 stricture, microbiome, enteritis

26

27 


\section{Background}

29

30

31

32

Over 3.5 million people worldwide are affected by inflammatory bowel disease (IBD), a chronic gastrointestinal (GI) inflammatory disease triggered by interactions between host, microbes and the environment ${ }^{1-5}$. Two common forms of IBD are Crohn's disease (CD), which affects the small and large intestines, and ulcerative colitis (UC), which localizes to the large intestine. Over 200 genomic loci may confer increased IBD risk, with many of these genes associated with regulating host-microbe interactions ${ }^{1}$. The human GI tract harbors trillions of microorganisms from at least 400 species that compose the intestinal microbiota ${ }^{6,7}$. In healthy individuals, the microbiome influences many physiological functions such as extracting nutrients, maintaining the gut mucosal barrier, training immune cells and protecting against pathogens ${ }^{8}$. Dysbiosis occurs due to loss of beneficial microbes, expansion of pathobionts (opportunistic microbes), or reduction of microbial diversity. Dysbiosis has been associated with human diseases, including irritable bowel syndrome, obesity, psoriasis, rheumatoid arthritis, autism spectrum disorders, Clostridioles difficile infection and $\mathrm{IBD}^{8,9}$. Changes in the intestinal microbiota observed in IBD patients have included reduction of short chain fatty acid (SCFA) producing bacteria, reduced alpha diversity, decreased Firmicutes abundance, and increased abundance of facultative anaerobes, Proteobacteria and Bacteroidetes ${ }^{2-}$ $4,10-12$.

In captive common marmosets, GI diseases are the most common and widespread clinical finding ${ }^{13,14}$. IBD prevalence is reported to be as high as $28-60 \%$ in captive marmosets and presents with diarrhea, weight loss, enteritis, muscle atrophy, alopecia, hypoproteinemia, anemia, elevated liver enzymes, and a failure to thrive ${ }^{13,15}$. The IBD diagnosis can be refined to chronic lymphocytic enteritis (CLE) with histologic findings, such as small intestinal localization, shortened villi, crypt epithelial hyperplasia, and lymphocytic infiltration of the lamina propria ${ }^{13,14}$. Potential marmoset 
51 biomarkers include calprotectin and matrix metalloproteinase $9^{16,17}$, but clinical interventions

52 involving glucocorticoids, gluten-free diets, Giardia treatment, etc. have yielded mixed results ${ }^{18-}$

20. In addition to IBD, a novel chronic GI disease has been described in young adult marmosets

54 characterized by duodenal dilation or stricture near the major duodenal papilla ${ }^{21,22}$. Clinical signs,

55 such as diarrhea, weight loss, or poor weight gain, resemble IBD but increased vomiting is also

56 observed. This syndrome was associated with hypoalbuminemia, hypoglobulinemia,

57 hypoproteinemia, hypocalcemia (total), elevated alkaline phosphatase, anemia, and in some cases,

58 leukocytosis ${ }^{22}$. Histologically, duodenal mucosal ulcerations with associated chronic-active

59 granulocytic and lympho-histiocytic inflammation were observed.

As the microbiome has been associated to human GI diseases, factors affecting the microbiome in non-human primates (NHP) are being explored, such as species, social structure, environment and $\operatorname{diet}^{23-26}$. Captivity and captive diets have been associated with microbial diversity loss, shifts in

63 the Firmicutes:Bacteroidetes ratio, and increased GI disease and mortality ${ }^{23,26,27}$. Dietary

64 specialists, such as marmosets, are more susceptible to captivity-associated dietary changes ${ }^{26}$.

65 Marmosets are exudivores that consume large amounts of indigestible oligosaccharides from tree 66 gums $^{28}$, and may harbor specific gut microbes dedicated to carbohydrate metabolism. Currently,

67 few reports on the marmoset microbiome are available ${ }^{29-34}$. In this study, we evaluated microbiome, serum chemistry and complete blood count (CBC) samples from healthy marmosets

$69(n=91)$ and marmosets with $\operatorname{IBD}(n=59)$ or duodenal ulcer/strictures $(n=23)$, collected during 70 physical examinations or necropsies over a two-year period. 'Healthy' controls were defined as

71 individuals not clinically diagnosed with IBD or strictures and not receiving chronic drug 72 treatments during the study period. Unique microbial profiles were associated with the four sources 73 that populated the MIT colony. We identified changes in both microbial communities and blood 
74 parameters that may serve as marmoset biomarkers for IBD and strictures, and propose that

75 marmosets may be useful animal models to study CD and Clostridium-driven GI disorders, such

76 as duodenal strictures.

\section{$77 \quad$ Results}

78

79

80

81

82

\section{Microbial Diversity in the Intestinal Microbiota of the Common Marmoset.}

303 samples from 91 healthy marmosets were analyzed to determine the normal microbiota (Table 1). $99 \%$ of the average microbial abundance in feces was captured by Bacteroidetes, Firmicutes, Proteobacteria, Fusobacteria and Actinobacteria (Fig. 1a). The microbiome profile observed in healthy, MIT marmosets resembles the microbiome observed in human stool with dominance of the phylum Bacteroidetes (average 63.2\%), followed distantly by Firmicutes and Proteobacteria ${ }^{7}$. As observed in humans ${ }^{7}$, Bacteroidetes abundance varied significantly, ranging from 8-86\%. Bacteroidetes were predominantly represented by Bacteroides, Prevotella 9 and Parabacteroides. The most abundant Firmicutes were Megamonas, Megasphaera and Phascolartcobacterium. Anaerobiospirillum, Sutterella and Escherichia-Shigella were the most common Proteobacteria. Notably, Bifidobacterium were present in low abundance compared to other reported marmoset microbiomes $^{29,30}$ (Supp. Table 1).

\section{Source population impacted microbiome diversity}

Having established the baseline microbiome for healthy, MIT marmosets, we explored the effects of age, sex and original source, and found that source strongly influenced composition (Fig 1b). MIT's colony originally received marmosets from four sources (A, B, CLEA and NEPRC), which we designated $\mathrm{MIT}^{\mathrm{A}}$, $\mathrm{MIT}^{\mathrm{B}}$, MIT ${ }^{\mathrm{CL}}$ and $\mathrm{MIT}^{\mathrm{NE}}$ following importation. Marmosets were housed in two buildings and provided standardized diet, husbandry and veterinary care. For this study, 
marmosets were co-housed with same-source animals. Using multiple estimators for alpha

97 diversity, we noted that species richness estimators significantly differed between healthy marmosets by source, but not sex or age. $\mathrm{MIT}^{\mathrm{NE}}$ marmosets had higher observed OTUs and Chaol

99 values compared to other sources ( $\mathrm{P}<0.001$ vs. each source, both metrics) (Fig 1c, Supp. Fig 1). $\mathrm{MIT}^{\mathrm{B}}$ had significantly higher alpha diversity compared to $\mathrm{MIT}^{\mathrm{CL}}$ (observed OTUs, $\mathrm{P}<0.05$; Chao1, $\mathrm{P}<0.01)$ and $\mathrm{MIT}^{\mathrm{A}}(\mathrm{Chao1}, \mathrm{P}<0.05)$. However, differences were not observed when accounting for evenness (Shannon diversity or Pielou's evenness). Clustering of samples based on source (Unweighted UniFrac: PERMANOVA, P<0.001; beta-dispersion, NS) (Fig 1d), but not sex or age, was also observed (Supp. Fig 2).

\section{Bacteroides and Prevotella Define Microbial Communities of Sources}

We next identified 63 differentially abundant genera between the 4 sources in the lower gut using ANCOM (Analysis of Composition of Microbiomes). 13 genera were present at relative abundances greater than 1\% in at least one source (Supp. Table 2). High abundance of Bacteroides characterized $\mathrm{MIT}^{\mathrm{NE}}$ and $\mathrm{MIT}^{\mathrm{B}}$ samples, while $\mathrm{MIT}^{\mathrm{CL}}$ and $\mathrm{MIT}^{\mathrm{A}}$ were primarily colonized by genus Prevotella 9 (Fig. 1b). The Bacteroidaceae:Prevotellaceae ratios for $\mathrm{MIT}^{\mathrm{A}}, \mathrm{MIT}^{\mathrm{CL}}, \mathrm{MIT}^{\mathrm{B}}$, and $\operatorname{MIT}^{\mathrm{NE}}(0.44,0.39,1.23$ and 2.17 , respectively) emphasize source-associated differences

112 reflected in these two genera. Anaerobiospirillum, another highly abundant genus, represented 8.5-

$113 \quad 13.8 \%$ of bacterial in three sources but had low numbers in $\mathrm{MIT}^{\mathrm{CL}}$ marmosets (1.5\%).

114 Next, we explored the ability of classification models to identify marmoset source based on 115 microbiome data (Supp. Fig 3a). After evaluating multiple models, we developed random forest 116 (RF) classification models to identify healthy marmosets by source. After ranking ASVs based on

117 their importance to the model, we iteratively created new models to determine the minimum 118 number of ASVs required to achieve stability in accuracy, and selected an optimized model using 
10 ASVs (Supp. Fig 3b, 3c). The optimized model achieved an accuracy of $93 \%$ with $100 \%$ sensitivity and 95\% specificity. The RF model confirmed that despite importation and assimilation, unique source-specific signature microbiota were retained by cohousing same-source animals.

\section{Prevalence of GI disease in MIT-housed Marmosets}

To study the effects of GI disease on the microbiome, marmosets were categorized as healthy $(n=91)$, IBD $(n=59)$ or duodenal stricture $(n=23)$. Strictures were mainly observed in MIT ${ }^{\mathrm{NE}}(21$ of 23 cases) with a $26 \%$ prevalence in this cohort. IBD was observed throughout the colony with varied prevalence (MIT ${ }^{\mathrm{CL}}, 55 \%$; $\mathrm{MIT}^{\mathrm{NE}}, 29 \%$; $\mathrm{MIT}^{\mathrm{A}}$, 27\%; and $\mathrm{MIT}^{\mathrm{B}}, 22 \%$ ) (Supp. Table 3).

\section{Effects of Duodenal Strictures on the microbiome and blood analysis}

As strictures predominantly affected $\mathrm{MIT}^{\mathrm{NE}}$, we next investigated the effects of duodenal strictures on the marmoset microbiome in this cohort. "Progressors," marmosets that had or developed strictures, had markedly different microbiomes compared to "non-progressors," animals that remained healthy or developed other diseases (Fig. 2a, Supp. Fig. 4a). On average, a 32\% decrease in Bacteroides was observed in stricture cases (35.8\% abundance in non-progressors vs. $24.5 \%$ in progressors), which decreased the Bacteroides:Prevotella 9 ratio from 3.1 in non-progressors to 1.4 in progressors. Anaerobiospirillum, the second most abundant genus in non-stricture marmosets $(13 \%)$, decreased to $4.6 \%$ in stricture cases. Concurrently, a $50 \%$ increase in Megamonas was observed in progressors (Fig. 2a). ANCOM identified Anaerobiospirillum and Clostridium sensu stricto 1 as differentially expressed, with Clostridium sensu stricto 1 increases measured in progressors.

Despite changes in microbial composition, no changes in alpha diversity were observed using multiple metrics. We then optimized a 9 ASV RF model that minimized the number of ASVs while 
141

142

143

144

145

146

147

148

149

150

151

152

153

154

155

156

157

158

159

160

161

162

163 maximizing accuracy of stricture classification (Fig. 2b, Supp. Fig. 4b, 4c). Of these 9 ASVs, 3 Anaerobiospirillum ASVs, as well as Bacteroides and Parabacteroides ASVs, decreased in progressors. Increases were observed in ASVs from Bifidobacterium, Clostridium sensu stricto 1, Oribacterium, and Megamonas. The receiver operating characteristic (ROC) curve for this model had an area under the curve (AUC) of 0.82 (Fig. 2c) with an accuracy of $85 \%$, a sensitivity of $100 \%$ and a specificity of $45 \%$.

As ANCOM and our model highlighted the role of Clostridium sensu stricto 1, we investigated its presence in strictures. Clostridium sensu stricto 1 encompasses Clostridium species $C$. tetani, $C$. botulinum, C. kluyveri, C. acetobutylicum, C. novyi, C. perfringens and C. beijerinckii, which are considered pathogenic and indicate less healthy and less diverse microbiota ${ }^{35}$. Using representative sequences assigned to Clostridium sensu stricto 1 ASVs, we determined that 232,156 (69\%) reads shared $>99 \%$ identity over the 370 bp sequence with $C$. perfringens. Remaining reads matched with C. baratii (19\%), C. colicanis (7\%) and unknown Clostridium species (6\%). Importantly, ASV256, which increased 6-fold in progressors, shared 100\% identity with $C$. perfringens. We then sought to confirm the presence of this organism by culture and 16S rRNA Sanger sequencing of clinical isolates. C. perfringens was isolated in 4 of 9 duodenum samples from marmosets with histologically confirmed strictures. C. baratii or $C$. sardiniense, a rare causative agent of botulism $^{36}$, was isolated from 1 of 9 samples. As the pathogen was isolated at the stricture site, we analyzed the microbiome in duodenal samples from stricture $(\mathrm{n}=17)$ and non-stricture cases $(\mathrm{n}=12)$ to determine if increased gut abundance reflected a duodenal infection. Clostridium sensu stricto 1 was observed at greater than $1 \%$ abundance in $76 \%$ of strictures $(13 / 17)$ but only in $16 \%$ of nonstricture cases (2/12). In 8 stricture cases, the bacterium was the most abundant genus with abundances ranging from $37-87 \%$. Interestingly, one non-stricture sample with $30 \%$ abundance of 
Clostridium sensu stricto 1 had duodenal pathology characterized by mild duodenal mucosal congestion (Fig 2d).

Next, we developed RF models using serum chemistry profiles or CBC data to categorize stricture amylase) classified progressors and non-progressors with $84.8 \%$ accuracy, a sensitivity of $76.5 \%$,

Fig. 4g-i). All variables, except RDW, decreased in strictures. Of note, weight was excluded from these models as severe weight loss is observed, which masked the contribution of other parameters. Exclusion of weight data did not decrease the predictive power of serum chemistry or CBC models.

\section{Effects of IBD on the microbiome and blood analysis}

177 While strictures were observed predominantly in $\mathrm{MIT}^{\mathrm{NE}}$, IBD was diagnosed in the four sources. remained healthy or developed non-IBD diseases. Across the colony, microbiome richness decreased in IBD progressors (Chao1, $\mathrm{P}<0.001$; Observed OTUs, $\mathrm{P}<0.001$ ) but these changes were not observed when accounting for evenness (Shannon's index and Pielou's evenness) (Fig. 3a). individual microbes were consistently associated with IBD across all sources. Even in disease, 
PC1 position were significantly different between healthy and IBD cases in the entire dataset (All, $\mathrm{P}<0.001)$, and in 3 of 4 sources $\left(\mathrm{MIT}^{\mathrm{B}}, \mathrm{P}<0.01 ; \mathrm{MIT}^{\mathrm{CL}}, \mathrm{P}<0.001 ; \mathrm{MIT}^{\mathrm{A}}, \mathrm{P}<0.05 ; \mathrm{MIT}^{\mathrm{NE}}, \mathrm{P}=0.6\right)$ (Fig 3b). While no single dysbiotic IBD state existed, source-specific, healthy states could become source-specific, IBD states through similar perturbations of the microbiome.

To identify IBD-associated patterns observed in the dataset and within source-specific subsets, we examined ASVs correlated with PC1. Five Prevotellaceae ASVs (Prevotella 9 and unclassified genera) and 3 Megasphaera ASVs were positively correlated with PC1, while 5 Parabacteroides ASVs and 3 Bacteroides ASVs were anti-correlated to PC1. Due to their importance in our analysis and the human gut microbiome 7,38 , we examined the relationship between Bacteroides and Prevotella 9 in marmoset IBD. Using BLAST, 99.93\% of Prevotella 9 reads matched P. copri with a $>99 \%$ identity. Bacteroides reads matched multiple species including B. plebeius (48.3\%), B. vulgatus (16.8\%), B. uniformis (6.4\%), B. dorei (4.3\%), B. massiliensis (3,2\%), B. thetaiotaomicron $(1.9 \%)$, B. ovatus $(1.9 \%)$ and B. coprocola (1\%). Decreases in Bacteroides were observed in IBD progressors, while Prevotella 9 remained level or increased (Fig. 3c). Overall, the ratio of average Bacteroides abundance to average Prevotella 9 abundance was 1.83 in nonprogressors and 1.07 in IBD progressors, yielding a non-progressor/progressor ratio of 1.7. Similar non-progressor/progressor ratios were observed in all subsets, implying increased $P$. copri levels relative to Bacteroides spp. in marmoset IBD (Fig. 3c). We also developed 4 RF models to classify progressors and non-progressors using data from the entire colony, $\mathrm{MIT}^{\mathrm{B}}, \mathrm{MIT}^{\mathrm{CL}}$ and $\mathrm{MIT}^{\mathrm{NE}}$ (MIT ${ }^{\mathrm{A}}$ excluded due to insufficient $\mathrm{n}$ ). The top 25 ASVs in each model were compared, and 8 ASVs shared by at least 3 models were identified (Supp. Table 4). ASVs belonged to Sutterella (3), Megamonas (2), Bacteroides, Asteroleplasma, and Prevotella 9. Overlap in genera was determined by collapsing the 4 lists of ASVs by genus. Half of these ASVs belonged to 5 genera: 
Bacteroides $(\mathrm{n}=20)$, Sutterella $(\mathrm{n}=10)$, Megamonas $(\mathrm{n}=8)$, Bifidobacterium $(\mathrm{n}=7)$ and Prevotella $9(\mathrm{n}=7)$. These results suggest that shifts in Bacteroides, $P$. copri, Megamonas and Sutterella are observed in IBD progressors relative to source-specific, healthy states.

Unlike the microbiome data, source-dependent clustering was not observed in serum chemistry or CBC PCA plots of IBD marmosets (Supp. Fig. 5b-c). Therefore, RF classifiers were trained solely on IBD status. The serum chemistry RF model used 7 variables (calcium, GGT, albumin, A:G ratio, amylase, cholesterol, and alkaline phosphatase), and had an accuracy of $77 \%$, a sensitivity of $79 \%$, a specificity of $76 \%$ and AUC of 0.85 (Fig. 3d, Supp. Fig. 5d). The optimized CBC RF model used HGB, RBC, RDW, MPV and neutrophil percentage, and had an accuracy of 77\%, a sensitivity of $73 \%$, a specificity of $83 \%$ and AUC of 0.81 (Fig. 3d, Supp. Fig. 5e). In these models, calcium, hemoglobin and RBC were the most important in classifying IBD.

\section{Effects of GI disease on gene expression of the small intestine}

We tested whether strictures or IBD significantly altered marmoset transcriptomic profiles using RNA sequencing (RNAseq) on samples from IBD $(n=3)$ or stricture $(n=3)$ marmosets. Marmosets with strictures presented with gross thickening, duodenal stricture or ulceration $(0.5-1 \mathrm{~cm}$ aboral to the major duodenal papilla). Duodenal tissue evaluated was immediately distal to the lesion ("stricture") or in an equivalent anatomic region in IBD animals ("non-stricture"). While IBD animals served as non-stricture controls, thickened intestines were observed grossly, and duodenitis was noted. As the most affected intestinal site in $\mathrm{IBD}^{13}$, we selected the jejunum to evaluate IBD effects. Unlike the IBD duodenum, the jejunum of stricture cases presented minimal pathology 22 , and were used as “non-IBD," jejunum controls.

Comparing stricture and non-stricture duodenums, we identified 1,183 differentially expressed genes (DEG) (FDR <0.05) (Fig 4a, Supp. Table 5). To perform Gene ontology (GO) analysis, 
marmoset genes with official names were matched to Homo sapiens genes to retrieve Entrez IDs with associated GO categories. Analysis of this gene subset identified 903 DEGs with GO annotations. The top 15 biological processes (BP) with significant enrichment are listed in Table 2 (complete list - Supp. Table 6). Stricture samples enriched BP sets involved with intestinal absorption, and lipid metabolism, localization and transport (Fig 4b, Supp. Fig. 6a). Stricture upregulated genes encompassed cholesterol-associated genes including apoliproteins ( $A P O B$, $A P O A 1$ and $A P O A 4)$, transport genes (ABCG5, ABCG8, GRAMD1B, and STARD3), metabolic genes (DGAT1, CYP11A1, and CYP27A1) and binding/absorption genes (SOAT2, NPC1L1 and $S C A R B 1)$ (Supp. Table 5a). Other lipid-associated genes upregulated by stricture included genes associated with fatty acid binding proteins ( FABP1 and $F A B P 2)$, peroxisomes (PPARA, $A B C D 1$, $A C A A 1$ and $E P H X 2)$, ketogenesis (HMGCS2) and lipid synthesis (GPAM, SREBF1, SCAP, and $A C A C B)$. Enriched cellular membrane GO sets shared these lipid-associated genes due to functional overlap (Supp. Table 6, Supp. Fig. 6b). Interestingly, immunity-associated genes were more highly expressed in non-stricture duodenums (Fig 4b, Supp. Fig. 7), possibly due to the enteritis observed in IBD marmosets. These genes included antimicrobial responses $(L C N 2, L Y Z$, MUC20), toll-like receptors (TLR2 and TLR4), superoxide-generating NADPH oxidase activity (NOX1 and DUOX2), killer cell lectin-like receptor genes (KLRB1, KLRC1, KLRD1, and KLRF1), and chemokine activity and receptor binding (CXCL1, CXCL10, TFF2 and PF4) (Supp. Table 5b). The transcriptional profile implies the activity of natural killer (NK) cells, neutrophils and MHC class I protein complex binding.

1,984 DEGs were identified when comparing jejunums from IBD and non-IBD marmosets (Fig 4c, Supp. Table 7) following the exclusion of an IBD sample that did not cluster with other samples (Supp. Fig. 8). GO annotations were assigned to 1,586 DEGs, and the top 15 BP are 
T cell activation, adaptive immune responses, and regulation of immune response (Fig 4d, Supp.

Fig. 9). As observed in the non-stricture duodenum, genes associated with killer cell lectin-like TBX21, CD27, PTPRC, and IL18R1) had increased expression in IBD samples. (Supp. Table 7). In non-IBD animals, top GOs associated with homeostatic functions, such as synaptic signaling, development, and muscle contraction (Table 3b, Supp. Fig. 10).

\section{Discussion}

268 GI diseases are the most prevalent clinical disease in captive common marmosets ${ }^{13,14,39}$, but the affects bacterial composition, reduces alpha diversity, and alters host responses to disease ${ }^{23,26,40}$.

271 In captivity, NHP microbiomes lose distinctive, wild microbiota and become dominated by

272 Prevotella and Bacteroides, the most abundant genera in the modern human gut microbiome ${ }^{7,23,38}$.

273 In the largest marmoset microbiome study to date, our data supports the hypothesis that captivity

274 humanizes the primate microbiome, as Bacteroides and Prevotella 9 were the most abundant

275 genera with levels similar to those observed in human feces ${ }^{7,38}$. In humans, Prevotella and

276 Bacteroides abundances are anticorrelated, signifying that competitive advantages in metabolism

277 determine the dominant bacteria ${ }^{41,42}$. Prevotella increases have been associated with high-fiber, 
279 Westernized populations with diets rich in animal fat and protein ${ }^{41,42}$. Diets influence levels of

280 fibers, fermentation products, SCFA and bile acids (BA), which determine bacterial

281 communities $^{42}$. As our marmosets were fed a standardized diet, dietary differences cannot account

282 for the Prevotella- and Bacteroides-dominant profiles observed stably in our colony. Most bacteria

283 observed were acetate- or propionate-producers, such as Bacteroides, Prevotella,

284 Anaerobiospirillum, Phascolarctobacterium, Megamonas, and Megasphaera, with a low

285 abundance of butyrate producers, such as Lachnospiraceae ${ }^{43}$. However, Megasphaera has been

286 known to produce butyrate under specific conditions ${ }^{33,44}$. Inter-institutional differences greatly

287 affect marmoset microbiomes, as previous studies report marmoset gut microbiota dominated by

288 Actinobacteria $^{29,30}$, Firmicutes ${ }^{33,34}$, Proteobacteria ${ }^{24,45,46}$ and Bacteroidetes ${ }^{31,32,46}$. At the

289 Biomedical Primate Research Centre (BPRC) (Rijswijk, the Netherlands), Actinobacteria,

290 represented by Bifidobacterium and Collinsella, was the most abundant phylum (66\%), while

291 Bacteroides and Prevotella represented $<5 \%$ of the microbiome $\operatorname{each}^{29}$. BPRC marmosets have

292 access to outdoor and indoor enclosures, as well as food enrichment, such as insects and gums,

293 several times a week ${ }^{29}$. We hypothesize that increased environmental exposure and enrichment

294 promotes a wild-like microbiome, rich in bifidobacteria that help metabolize oligosaccharide-rich

295 tree gums, a common food source for wild marmosets ${ }^{45,47}$. High abundances of Actinobacteria are

296 observed in wild callitrichids, but not in captive and semi-captive marmosets ${ }^{24}$. Unexpectedly,

297 Ross et al. also reported high Bifidobacterium levels in marmosets housed within a specific-

298 pathogen free (SPF) barrier facility and at the Southwest National Primate Resource Center

299 (SNPRC) $)^{30}$. In contrast to the BPRC, SPF marmosets fed exclusively irradiated feed, nuts, seeds and dried fruits had median Bifidobacterium abundances of $17 \%{ }^{30}$. This was much higher than the

301 non-SPF parent colony at SNPRC, which had median Bifidobacterium frequencies of $4 \%$ and high 
302 levels of Fusobacterium ${ }^{30}$. However, a follow-up report from the barrier facility showed bacterial

303 shifts with an increased Bacteroidetes abundance (35\%) and a slight decrease in

304 Bifidobacteriaceae $(12 \%)^{31}$. Similar to our study, few age-related changes in the microbiome were

305 observed $^{31}$. In a colony with a microbiome similar to the MIT profile, microbiome synchronization

306 occurred within a year in imported marmosets, characterized by expansion of Bacteroidetes ${ }^{46}$.

307 Imported cohorts retained unique features following microbiome synchronization ${ }^{46}$, supporting

308 our findings that source-specific microbiomes persist despite standardization of husbandry and

309 diet. These studies demonstrate that inter-institutional differences can promote stable microbiomes

310 in clinically healthy animals across a large range of bacterial compositions. In other NHP, wild-

311 like microbiota may prevent captivity-associated illnesses ${ }^{23}$. The resilience to perturbations of

312 different bacterial compositions in marmosets is unknown. Understanding and manipulating the

313 marmoset microbiome may help prevent disease, and due to their importance as research models

314 in neuroscience, aging, and toxicology, having marmosets with "humanized" microbiota may

315 better represent the human condition.

316 In this study, we evaluated a marmoset colony with a "humanized" microbiota 48 and compared the

317 microbiota of clinically healthy individuals with marmosets with two GI diseases. While captivity

318 increases susceptibility to GI disease, we observed source-specific differences in disease

319 prevalence. MIT $^{\mathrm{NE}}$ marmosets had the highest Bacteroidaceae abundance (37\%) and the lowest

320 Prevotellaceae levels (17\%), and were most susceptible to strictures, a novel GI disease in

321 marmosets ${ }^{21,22}$. This duodenal syndrome was found in $21.9 \%$ of necropsy cases in an institution ${ }^{21}$,

322 while MIT $^{\mathrm{NE}}$ marmosets had a $26 \%$ prevalence. Clinical signs include vomiting, bloating, weight

323 loss and a palpable thickening of the duodenum that can be visualized through radiography and

324 ultrasound ${ }^{21,22}$. Stricture-associated dysbiosis featured reductions in Bacteroides and 
Anaerobiospirillum, and Megamonas increases. Our analysis of strictures highlighted the importance of decreases in Anaerobiospirillum and increases in Clostridium sensu stricto 1. While Anaerobiospirillum has been previously reported in healthy marmosets, dogs and cats ${ }^{45,49}$, these bacteria may cause GI disease in humans ${ }^{49}$. However, Anaerobiospirillum was present in high abundances in our healthy marmosets, and reduced levels were seen in disease.

As $C$. perfringens was detected at higher levels in the duodenal lesions of diseased animals by culture and sequencing, we propose that $C$. perfringens is a potential causative agent of duodenal disease in marmosets. $C$. perfringens is a known GI pathogen that can encode multiple toxins (alpha, beta, epsilon, iota, perfringolysin $\mathrm{O}$, and enterotoxin) ${ }^{35}$. In marmosets and other NHP, $C$. perfringens can cause gas gangrene and gastric dilatation syndrome ${ }^{50-52}$. Of note, C. perfringensinduced gas gangrene was reported in the institution that first reported duodenal strictures ${ }^{50}$. In the United Kingdom, $C$. perfringens is one of the top 5 causes of foodborne death ${ }^{53}$, and has been linked to diarrhea, Clostridial necrotizing enteritis (CNE), necrotizing enterocolitis (NEC), UC and enterotoxemia in humans and other mammals ${ }^{35,54}$. CNE is a necrotizing inflammation of the small intestine that can induce mild diarrhea or severe abdominal pain, vomiting and ulcers ${ }^{35}$. NEC predominantly affects infants due to intestinal immaturity or dysbiosis ${ }^{35,54}$. While these symptoms match the clinical presentation of duodenal strictures in marmosets, they are non-specific. However, intestinal strictures developed in 11-29.5\% of NEC infants in both small and large intestines and could occur up to 20 months post-diagnosis ${ }^{55,56}$. Based on the site of $C$. perfringens infection at the junction of the duodenum and the common bile duct, we hypothesize that BA deregulation due to dysbiosis or antibiotic treatment may facilitate $C$. perfringens infection. Antibiotic usage in infants has been linked with increased NEC risk $^{57}$, and antibiotics are commonly prescribed to treat NHP GI diseases. Furthermore, C. perfringens was overrepresented 
in dogs with chronic enteropathy, an IBD-like disease, and bacterial abundance was regulated by secondary BAs, deoxycholic acid and lithocholic acid, that are produced by gut bacteria ${ }^{58,59}$.

In addition to the role of $C$. perfringens, our serum chemistry and $\mathrm{CBC}$-based RF models were highly sensitive in classifying strictures. Decreased total protein levels are often observed with GI disease and may indicate poor digestion/absorption. The importance of amylase and lipase in our stricture model is supported by clinical findings of cholecystitis and secondary pancreatitis ${ }^{22}$. Secondary pancreatitis, attributed to extension from the duodenal ulcer, was observed in 15 of 17 cases scored ${ }^{22}$. In the CBC-based model, HCT, HGB, RBC, RDW, and MCH relate to red blood cell function and suggested anemia. Anemia, a common finding in marmosets with strictures and $\mathrm{IBD}^{15,22}$, is also a risk factor for NEC in humans ${ }^{60}$. Interestingly, transcriptomic analysis of strictures showed enrichment of lipid metabolism and intestinal absorption genes, which may reflect enterocyte damage and is consistent with lipidomic alterations induced by $C$. perfringens alpha-toxin, a phospholipase $\mathrm{C}^{61}$. Increased expression of $F A B P 1$ and $F A B P 2$ was observed. These genes encode for liver and intestinal fatty-acid binding proteins (LFABP and IFABP), respectively, and are often used as biomarkers of GI diseases, including $\mathrm{NEC}^{57}$. To our knowledge, correlations of gut $F A B P 2$ levels with serum IFABP levels have not been described, but we hypothesize that increased expression might be a compensatory mechanism triggered by enteritis. While increased inflammatory responses were not observed due to the lack of healthy control tissue, based on the C. perfringens infection, development of enteritis, anemia and strictures and deregulation of lipid metabolism, we believe marmosets could be developed as a model to investigate the mechanisms of bacterially-driven CNE/NEC.

In contrast, a unique microbial signature for IBD was not evident. Consistent with human studies, marmoset IBD decreased alpha diversity ${ }^{3,11,37}$. Human IBD is characterized by the loss of health- 
371 associated genera, such as Roseburia, Faecalibacterium, Eubacterium, Ruminococcus and

372 Subdoligranulum ${ }^{2,3,37,62}$, but these bacteria have not been found in high abundance in marmosets ${ }^{29-}$

373 . Other potentially beneficial taxa in humans that have been observed in marmosets include

374 Bifidobacterium, Bacteroides, Collinsella, and Phascolarctobacterium ${ }^{3,62}$. Increases in

375 Lactobacillus, Ruminococcus gnavus, Enterobacteriaceae, Pasteurellaceae, Veillonellaceae, and

376 Fusobacteriaceae have been associated with $\mathrm{IBD}^{3,37,62}$. While convergence to a single dysbiotic

377 IBD state was not observed, multiple, source-specific states were associated with IBD. Within

378 each source population, IBD progressors had higher average abundances of $P$. copri and

379 Megamonas, as well as decreased abundance of Bacteroides, relative to controls. Our RF models

380 also highlighted Sutterella, a bacteria associated with negative fecal microbiota transplantation

381 outcomes, shorter remission periods in UC patients ${ }^{63,64}$, and its ability to dampen immune

382 responses ${ }^{65}$. Megamonas, along with B. plebeius, deregulate BA metabolism in CD patients ${ }^{66}$,

383 which could cause dysbiosis and opportunistic pathogen infections. However, while Megamonas

384 increases were observed, Bacteroides decreased in marmoset IBD. Most Bacteroides reads

385 matched B. plebeius, a non-B. fragilis group species ${ }^{67}$. B. plebeius ASVs were the most abundant

386 in the two Bacteroides-dominated cohorts, and only $20 \%$ of Bacteroides reads matched members

387 of the B. fragilis group, the most frequently isolated and virulent species in clinical specimens ${ }^{68}$.

388 Furthermore, the role of the $B$. fragilis group in IBD is inconclusive, as they both modulate

389 immunity and cause infections ${ }^{3,68-70}$.

390 While the effects of Bacteroides and Prevotella spp. in IBD patients have not been

391 understood $^{3,71,72}$, Prevotella have been considered inflammophilic pathobionts, commensal

392 bacteria known to thrive in inflammatory environments and promote inflammatory diseases, such

393 as periodontitis, bacterial vaginosis, rheumatoid arthritis (RA), and metabolic disorders ${ }^{73-75}$. 
Prevotella, including P. copri, activate TLR2, elicit specific IgA and IgG responses and promote the release of IL-1, IL-8, IL-6, IL-17, IL-23, and CCL20, which leads to neutrophil recruitment, reduced T helper 2 (Th2) cells and induction of Th17 cells ${ }^{73-77}$. In the gut, Prevotella has been linked to diarrhea, HIV-induced gut dysbiosis, irritable bowel syndrome and more severe colitis ${ }^{78-}$ ${ }^{80}$. In a small study, higher levels of Prevotella were observed in marmosets with IBD compared to controls ${ }^{46}$. Furthermore, models of RA and colitis have shown that transfer of Prevotella- or $P$. copri-rich microbiota to mice transmitted disease phenotypes ${ }^{74,77,78}$. A possible mechanism could be linked to cycles of expansion and relaxation observed in $P$. copri abundance in healthy individuals, but absent in IBD patients ${ }^{5}$. Constant $P$. copri signals might promote chronic inflammation, but natural control of $P$. copri in the microbiome might prevent disease-causing chronic inflammatory states. In our study, IBD-associated enteritis upregulated pro-inflammatory immune responses in the duodenum and jejunum. Multiple genes associated with NK cell functions were upregulated by IBD, including genes associated with high cytolytic effector activity, cytotoxicity and IFN- $\gamma$ production $(C D 244, C D 160, I L 18 R 1, F Y N \text {, and } I F N G)^{81,82}$. In addition to $I F N G$, genes associated with Th1 cells (TBX21, CCR2, CCR5, and IL2RB) were also upregulated. In humans, killer immunoglobulin receptor (KIR) polymorphisms have linked NK cells with $\mathrm{CD}^{83}$. Further studies are needed to determine if $P$. copri causes enteritis and IBD in marmosets via NK cells.

This study is the largest evaluation of the captive marmoset microbiome, and is the first to systematically compare clinically healthy marmosets and marmosets with two GI disorders. The common marmoset may be a useful model to investigate $C$. perfringens-associated enteritis and intestinal strictures, as well as $P$. copri-mediated IBD. As observed in humans, a range of stable microbiome profiles may exist in clinically healthy marmosets. Better understanding of these 
417 profiles, the effects of diet and husbandry, and their inherent robustness to insults and disease will

418 be helpful in promoting animal health, developing better models of human disease and

419 understanding how to modulate microbial communities.

420 Materials and Methods

421 Animals.

422 Common marmosets (Callithrix jacchus) were housed at the Massachusetts Institute of

423 Technology in Cambridge, MA, from marmosets sourced from the New England Primate Research

424 Center (NEPRC), an international primate center (CLEA Japan Inc.), and two biotech companies

425 (A and B). Subsequently, the four sources will be referred to as MIT ${ }^{\mathrm{NE}}, \mathrm{MIT}^{\mathrm{CL}}$, MIT ${ }^{\mathrm{A}}$, and $\mathrm{MIT}^{\mathrm{B}}$.

426 All animals were housed in pairs or family groups within two vivaria at MIT, an AAALAC

427 International accredited facility. Of the animals evaluated in this survey, 85 were male and 88 were

428 female. All marmosets included in this study were on an animal use protocol approved by the MIT

429 Institutional Care and Use Committee (IACUC).

430 The animal holding room temperature was maintained at $74.0+/-2^{\circ} \mathrm{F}$ with a relative humidity of

$43130-70 \%$. The light cycle was maintained at a 12:12h light:dark cycle. Marmosets were housed in

432 cages composed of stainless-steel bars and polycarbonate perches with the following dimensions:

433 30" W x 32" D x 67" H). Each cage had a nest box made of polycarbonate attached the outside of

434 the cage. Other cage furniture present in the cages included hammocks, hanging toys, and

435 manzanita wood branches. Foraging enrichment in the form of dried acacia gum-filled branches

436 and forage board were provided weekly. Cages were removed for sanitization on a biweekly

437 rotation. 
All animals received a base chow diet of biscuits (Teklad New World Primate Diet 8794). Initially, biscuits were soaked in water for at least 20 minutes, but the practice was then changed to a pouron/pour-off soak only. About halfway through the two-year period encompassing this study, biscuit prep protocol reverted to the original practice of a 20 -minute soak to alleviate any concerns that soaking duration could be contributing to the development of duodenal ulcers. In addition to the base chow, a cafeteria-style supplemental offering of fruits, vegetables, and additional protein sources including hard-boiled eggs, mealworms, cottage cheese or ZuPreem (Premium Nutritional Products, Inc., Mission, KS).

On a semiannual basis, preventative health physical exams were performed on all colony animals. Rectal swabs and fecal samples were collected and screened for potentially pathogenic bacteria (including Salmonella spp., Shigella spp, beta-hemolytic E.coli, Klebsiella spp., and Campylobacter spp.) and parasites (including Enterobius spp., Entamoeba spp., Giardia spp., Taenia spp., and Cryptosporidium spp.). Intradermal testing for Mycobacterium tuberculosis was performed semiannually as well. All animals derived from progenitor stock were negative for squirrel monkey cytomegalovirus, Saimiriine herpesvirus 1, Saimiriine herpesvirus 2, and measles virus. Complete blood count and serum chemistry analysis were performed on an annual basis and during diagnostic workup of clinical cases. Hematology analysis was performed by the MIT DCM diagnostic laboratory using a HemaVet 950 veterinary hematology analyzer (Drew Scientific, Oxford, CT). Serum chemistry analysis was performed by Idexx Laboratories (Westbrook, ME). Serum chemistry and complete blood counts data were collected from the clinical records from the MIT colony. Fecal $(n=223)$ and rectal swab $(n=342)$ were collected from common marmosets (Callithrix jacchus) (n=565 samples, 173 individuals) between 2016-2018. 
461 Stricture samples containing duodenal tissue and duodenal contents were collected from animals

462 during necropsies performed by clinical veterinarians and veterinary pathologists.

463 Representative sections of major organs were collected, fixed in 10\% neutral buffered formalin,

464 embedded in paraffin, sectioned at $5 \mu \mathrm{m}$, and stained using hematoxylin and eosin (HE) for

465 scoring by a boarded veterinary pathologist. Stricture samples were flash frozen in vials containing

466 Brucella broth in $20 \%$ glycerol and frozen at $-80^{\circ} \mathrm{C}$. The tissues were thawed in an anaerobic

467 atmosphere $\left(10 \% \mathrm{CO}_{2}, 10 \% \mathrm{H}_{2}, 80 \% \mathrm{~N}_{2}\right)$, and were homogenized with freeze medium with tissue

468 grinders. The homogenate was divided into the following aliquots. For aerobic culture, the

469 homogenates were plated onto chocolate agar, blood agar, MacConkey agar, and Brucella Broth

470 medium containing $10 \% \mathrm{FCS}$. The plates were incubated at $37^{\circ} \mathrm{C}$ in $5 \% \mathrm{CO}_{2}$ for $24-48$ hours. For

471 anaerobic culture, the homogenates were plated onto pre-reduced Brucella Blood Agar plates

472 (BBL) and inoculated into thioglycollate broth. The cultures were incubated at $37^{\circ} \mathrm{C}$ in an

473 anaerobic chamber (Coy Lab Products) with mixed gas $\left(10 \% \mathrm{CO}_{2}, 10 \% \mathrm{H}_{2}, 80 \% \mathrm{~N}_{2}\right)$ for 48 hours.

474 For microaerobic culture to detect the growth of Helicobacter spp., the homogenates were plated

475 onto selective antibiotic impregnated plates $(50 \mu \mathrm{g} / \mathrm{ml}$ amphotericin $\mathrm{B}, 100 \mu \mathrm{g} / \mathrm{ml}$ vancomycin,

$476 \quad 3.3 \mu \mathrm{g} / \mathrm{ml}$ polymyxin B, $200 \mu \mathrm{g} / \mathrm{ml}$ bacitracin, and $10.7 \mu \mathrm{g} / \mathrm{ml}$ nalidixic acid) ${ }^{84}$ and Brucella Blood

477 Agar plates after passing through $0.65 \mu \mathrm{m}$ syringe filter. The plates were placed into a vented jar

478 filled with mixed gas $\left(10 \% \mathrm{CO}_{2}, 10 \% \mathrm{H}_{2}, 80 \% \mathrm{~N}_{2}\right)$ and incubated at $37^{\circ} \mathrm{C}$ for up to 3 weeks. The

479 plates were checked every 2-3 days for growth. Aliquots of the homogenates were also used for

480 DNA extraction. All bacterial strains isolated from the different culture conditions were identified

by 16 s rRNA sequencing.

482 
484 Fecal DNA was extracted using the DNeasy PowerLyzer PowerSoil Kit, and DNA was amplified 485 using universal primers of F515 (GTGYCAGCMGCCGCGGTAA) and R926

486

487

488

489

490

491

492

493

494

495

496

497

498

499

500

501

502

503

504

505 (CCGYCAATTYMTTTRAGTTT) to target the V4 and V5 regions of bacterial 16S rRNA fused to Illumina adaptors and barcode sequences as described previously. ${ }^{85}$ Individual samples were barcoded and pooled to construct the sequencing library, followed by sequencing with an Illumina MiSeq instrument to generate pair-ended $300 \times 300$ reads. Sequencing quality was inspected using FastQC $^{86}$. Reads were processed using QIIME 2-2018.6 within the MicrobiomeHelper v. 2.3.0 virtual box ${ }^{85,87}$. Briefly, primer sequences were trimmed using the cutadapt plugin $^{88}$. Forward and reverse reads were truncated at 243 and 195 bases, respectively, prior to stitching and denoising reads into amplicon sequence variants (ASV) using DADA2. Samples with fewer than 7,500 reads were excluded. ASVs present in fewer than 3 samples and with less than 24 counts were also excluded. A total of 1085 ASVs were retained after filtering. Taxonomic classification was assigned using the custom 16S V4/V5 region classifier based on the SILVA 132 database (SSU Ref NR 99) ${ }^{89}$. Phylogenetic trees, composition, alpha rarefaction, beta diversity metrics and ANCOM (Analysis of Composition of Microbiome) ${ }^{90}$ were evaluated using built-in QIIME2 functions ${ }^{91}$. Microsoft Excel and R (v 3.6.3 at http://www.R-project.org/) were used to perform statistical analyses and graphically represent data. Additionally R libraries ggplot2 (2.2.1 $)^{92}$, caret $^{93}$, vegan ${ }^{94}$, pROC $^{95}$, and gtools ${ }^{96}$ were used to model microbiome data. Classifiers were trained on $80 \%$ of the samples and the discovered signatures were used to predict the populations on the remaining $20 \%$ of samples (testing). We analyzed the Bacteroides/Prevotella abundance ratio by taking the ratio of the averaged Bacteroides abundance and the averaged Prevotella abundance. 
Tissues were collected from the duodenum and jejunum from marmosets with either stricture or IBD during necropsies performed by clinical veterinarians and veterinary pathologists. In stricture cases, duodenal samples were distal of the site of stricture $(n=3)$, and in IBD cases, the same region

511 of the duodenum presented with mild thickening based on gross observations $(n=3)$. In IBD cases,

512 the jejunum presented with increased thickening $(n=3)$, while in stricture cases, the jejunum was 513 grossly normal $(n=3)$. Tissues were flash frozed in liquid nitrogen and stored at $-80^{\circ} \mathrm{C}$. RNA was 514 extracted using TRIzol reagent according to manufacturer's instructions (Thermo Fisher 515 Scientific). Total RNA was shipped on dry ice to Arraystar, Inc. (Rockville, MD) for quality control, rRNA depletion and sequencing on an Illumina HiSeq4000. FASTA files and the NCBI

517 RefSeq GTF files for Callithrix jacchus based on the March 2009 (WUBSC 3.2/calJac3) assembly

518 were obtained from the UCSC Genome browser ${ }^{97}$. Raw sequencing reads were mapped to an index

519 built from $C$. jacchus FASTA files using Rsubread ${ }^{98}$. Feature counts were obtained from the bam 520 files using annotated exons in the $C$. jacchus GTF files. Analysis was then performed using 521 edge $\mathrm{R}^{99,100}$. Lowly expressed exons were removed using a cutoff of 10 counts per million (CPM).

522 Normalization was performed using the Trimmed Mean of M-values (TMM) method.

523 Multidimensional scaling (MDS) plots and heatmaps were used to evaluate grouping of biological

524 samples. Data was fitted using the glmQLFit function that uses a generalized linear model (GLM)

525 implementing a quasi-likelihood (QL) fitting method. Quasi-likelihood F-tests were performed to 526 test for differential expression based on False Discovery Rate (FDR) adjusted P-values of 0.05. To 527 retrieve Gene Ontology (GO) classifications, C. jacchus genes that matched Homo sapiens gene 528 names were assigned both the C. jacchus and Homo sapiens Entrez IDs. GO analysis was 529 performed using limma ${ }^{101}$, AnnotationDbi ${ }^{102}, \mathrm{GO}^{\mathrm{db}} \mathrm{b}^{103}$, topGO $^{104}$, mygene ${ }^{105}$ and org.Hs.eg.db. 
530 Data was visualized using ggplot2, gplots, Rgraphviz ${ }^{106}$, colorspace ${ }^{107}$ and ggVennDiagram ${ }^{108}$.

531 Analysis of the IBD dataset demonstrated that the expression profile of one sample differed from

532 the remaining samples and was excluded from the analysis presented.

\section{Data availability}

534 RNAseq data is available under NCBI GEO accession number GSE156839. Microbiome data is 535 available under NCBI BioProject PRJNA659472.

536 Acknowledgements

537 This work was supported in part by a grant from the MIT McGovern Institute, NIH grant T32 538 OD010978 and by the National Institute of Environmental Health Sciences of the NIH under award 539 P30-ES002109.

540 Author contributions

541 Conception and design: AS, SCA, MAB, SM, JGF. Data acquisition, analysis and interpretation: 542 AS, SCA, MAB, JAM, MAL, JD, SM. Manuscript: AS, SCA, JGF

\section{Captions}

544 Figure 1. A) Gut microbiome profiles of healthy, common marmosets at the phylum level exhibit

545 a Bacteroidetes-dominant and human-like microbiome. B) Averaged relative abundances at the 546 genus level show differences associated with source but few differences based on sex or age. C) 547 Observed OTUs were increased in $\mathrm{MIT}^{\mathrm{NE}}$ vs. all sources and $\mathrm{MIT}^{\mathrm{B}}$ compared to MIT ${ }^{\mathrm{A}}$ and MIT ${ }^{\mathrm{CL}}$, 548 but metrics involving evenness, such as Shannon's diversity index, showed no difference. D) 549 PCoA plot using Unweighted UniFrac metric shows clustering of microbiome profiles based on marmoset source. ${ }^{*}, P<0.05 ; * *, P<0.01$ and $* * *, P<0.001$. 
551 Figure 2. A) Microbiome composition of samples at the genus level and pie charts with average

552 bacterial abundances of stricture progressors and non-progressors show dysbiosis associated with

553 stricture characterized by decreased Bacteroides and Anaerobiospirillum and increased

554 Megamonas. B) Nine ASVs identified by a random forest model that can correctly classify stricture

555 and non-stricture samples with a 85\% accuracy. C) Area under the curve (AUC) of receiver

556 operating characteristic (ROC) curves for random forest models using microbiome, serum

557 chemistry or complete blood count show strong performance of models in classifying strictures

558 and non-strictures. D) Relative abundance of Clostridium sensu stricto 1 reads in duodenal tissues

559 is increased in stricture cases compared to non-stricture cases.

560 Figure 3. A) Decreased richness was observed in IBD marmosets (Observed OTUs and Chao1) 561 compared to non-IBD marmosets similar to what is observed in humans. B) Increases in PC1

562 relative to source-specific, non-IBD controls were observed in 3 of 4 sources. C) Bacteroides and

563 Prevotella 9 levels are shown by source and IBD status. A lower overall and source-specific

564 Bacteroides:Prevotella 9 ratio is observed in IBD cases regardless of source-specific differences

565 in abundances of these two genera. D) AUC of ROC for random forest models using serum

566 chemistry and CBC show strong performance of models in classifying IBD progressors and non-

567 progressors. *,$P<0.05 ; * *, P<0.01$ and $* * *, P<0.001$.

568 Figure 4. A) Differentially expressed genes $(\mathrm{DEG})(\mathrm{FDR}<0.05)$ in the duodenum of non-stricture

569 and stricture cases. B) Gene ontology (GO) sets enriched in stricture cases show upregulation of

570 lipid metabolism, transport and localization. Non-stricture cases have enrichment of immune

571 processes, possibly due to underlying pathology caused by IBD. C) DEG (FDR <0.05) in the

572 jejunum of non-IBD and IBD cases. D) IBD samples are enrich GO sets associated with immunity

573 and immune cell activation. 
574 Table 1. Demographics of samples used classified by Sex, Age, Sample Type and Source. Columns

575 break down samples by disease status.

576 Table 2. Top Gene Ontology sets observed in RNAseq analysis of stricture progressors and non-

577 progressors

578 Table 3. Top Gene Ontology sets observed in RNAseq analysis of IBD progressors and non-

579 progressors 
600

601

602 603

604 605

606

607

608

609

610

611

612

613

614

615

616

617

\section{References}

1. Huang H, Fang M, Jostins L, et al. Fine-mapping inflammatory bowel disease loci to single-variant resolution. Nature. 2017;547(7662):173-178. doi:10.1038/nature22969

2. Morgan XC, Tickle TL, Sokol H, et al. Dysfunction of the intestinal microbiome in inflammatory bowel disease and treatment. Genome Biol. 2012;13(9). doi:10.1186/gb2012-13-9-r79

3. Gevers D, Kugathasan S, Denson LA, et al. The treatment-naive microbiome in new-onset Crohn's disease. Cell Host Microbe. 2014;15(3):382-392. doi:10.1016/j.chom.2014.02.005

4. Kostic AD, Xavier RJ, Gevers D. The microbiome in inflammatory bowel disease: Current status and the future ahead. Gastroenterology. 2014;146(6):1489-1499. doi:10.1053/j.gastro.2014.02.009

5. Lloyd-Price J, Arze C, Ananthakrishnan AN, et al. Multi-omics of the gut microbial ecosystem in inflammatory bowel diseases. Nature. 2019;569(7758):655-662. doi:10.1038/s41586-019-1237-9

6. Ley RE, Peterson DA, Gordon JI. Ecological and evolutionary forces shaping microbial diversity in the human intestine. Cell. 2006;124(4):837-848.

doi:10.1016/j.cell.2006.02.017

7. Huttenhower C, Gevers D, Knight R, et al. Structure, function and diversity of the healthy human microbiome. Nature. 2012;486(7402):207-214. doi:10.1038/nature11234

8. Rajilić-Stojanović M. Function of the microbiota. Best Pract Res Clin Gastroenterol. 2013;27(1):5-16. doi:10.1016/j.bpg.2013.03.006

9. Durack J, Lynch S V. The gut microbiome: Relationships with disease and opportunities for therapy. $J$ Exp Med. 2019;216(1):20-40. doi:10.1084/jem.20180448

10. Frank DN, St. Amand AL, Feldman RA, Boedeker EC, Harpaz N, Pace NR. Molecularphylogenetic characterization of microbial community imbalances in human inflammatory bowel diseases. Proc Natl Acad Sci U S A. 2007;104(34):13780-13785. doi:10.1073/pnas.0706625104

11. Willing BP, Dicksved J, Halfvarson J, et al. A pyrosequencing study in twins shows that gastrointestinal microbial profiles vary with inflammatory bowel disease phenotypes. Gastroenterology. 2010;139(6). doi:10.1053/j.gastro.2010.08.049

12. Martinez-Medina M, Aldeguer X, Lopez-Siles M, et al. Molecular diversity of Escherichia coli in the human gut: New ecological evidence supporting the role of adherent-invasive E. coli (AIEC) in Crohn's disease. Inflamm Bowel Dis. 2009;15(6):872-882. doi:10.1002/ibd.20860

13. Ludlage E, Mansfield K. Clinical care and diseases of the common marmoset (Callithrix jacchus). In: Comparative Medicine. Vol 53. ; 2003:369-382.

14. David JM, Dick EJ, Hubbard GB. Spontaneous pathology of the common marmoset 
627

628

629

630

631

632

633

634

635

636

637

638

639

640

641

642

643

644

645

646

647

648

649

650

651

652

653

654

655

656

(Callithrix jacchus) and tamarins (Saguinus oedipus, Saguinus mystax). J Med Primatol. 2009;38(5):347-359. doi:10.1111/j.1600-0684.2009.00362.x

15. Baxter VK, Shaw GC, Sotuyo NP, et al. Serum albumin and body weight as biomarkers for the antemortem identification of bone and gastrointestinal disease in the common marmoset. PLoS One. 2013;8(12):e82747. doi:10.1371/journal.pone.0082747

16. Nakashima E, Okano Y, Niimi K, Takahashi E. Detection of calprotectin and apoptotic activity in the colon of marmosets with chronic diarrhea. J Vet Med Sci.

2013;75(12):1633-1636. doi:10.1292/jvms.13-0257

17. Yoshimoto T, Niimi K, Takahashi E. Serum matrix metalloproteinase 9 (MMP9) as a biochemical marker for wasting marmoset syndrome. J Vet Med Sci. 2016;78(5):837-843. doi:10.1292/jvms.15-0675

18. Otovic P, Smith S, Hutchinson E. The use of glucocorticoids in marmoset wasting syndrome. J Med Primatol. 2015;44(2):53-59. doi:10.1111/jmp.12159

19. Schroeder C, Osman AA, Roggenbuck D, Mothes T. IgA-gliadin antibodies, IgAcontaining circulating immune complexes, and IgA glomerular deposits in wasting marmoset syndrome - PubMed. Nephrol Dial Transplant. 1999;14(8):1875-1880.

20. Kuehnel F, Mietsch M, Buettner T, Vervuert I, Ababneh R, Einspanier A. The influence of gluten on clinical and immunological status of common marmosets (Callithrix jacchus). J Med Primatol. 2013;42(6):300-309. doi:10.1111/jmp.12055

21. Mineshige T, Inoue T, Yasuda M, Yurimoto T, Kawai K, Sasaki E. Novel gastrointestinal disease in common marmosets characterised by duodenal dilation: a clinical and pathological study. Sci Rep. 2020;10(1):1-10. doi:10.1038/s41598-020-60398-4

22. Artim SC, Sheh A, Burns MA, Fox JG, Muthupalani S. Abstracts of Scientific Presentations 2019 AALAS National Meeting: P139 A Syndrome of Duodenal Ulceration with Strictures in a Colony of Common Marmosets (Callithrix jacchus). J Am Assoc Lab Anim Sci. 2019;58(5):607-726.

23. Clayton JB, Vangay $\mathrm{P}$, Huang H, et al. Captivity humanizes the primate microbiome. Proc Natl Acad Sci U S A. 2016;113(37):10376-10381. doi:10.1073/pnas.1521835113

24. Malukiewicz J, Cartwright RA, Dergam JA, et al. The Effects of Host Taxon, Hybridization, and Environment on the Gut Microbiome of Callithrix Marmosets. bioRxiv. Published online July 22, 2019:708255. doi:10.1101/708255

25. Hicks AL, Lee KJ, Couto-Rodriguez M, et al. Gut microbiomes of wild great apes fluctuate seasonally in response to diet. Nat Commun. 2018;9(1):1-18. doi:10.1038/s41467-018-04204-w

26. Frankel JS, Mallott EK, Hopper LM, Ross SR, Amato KR. The effect of captivity on the primate gut microbiome varies with host dietary niche. Am J Primatol. 2019;81(12). doi:10.1002/ajp.23061

27. Malukiewicz J, Cartwright RA, Dergam JA, et al. The Effects of Host Taxon, Hybridization, and Environment on the Gut Microbiome of Callithrix Marmosets. bioRxiv. 
675

676

677

678

679

680

681

682

683

684

685

686

687

688

689

690

691

692

693

694

Published online 2019. doi:10.1101/708255

28. Rylands AB, de Faria D. Habitats, feeding ecology, and home range size in the genus Callithrix. In: Rylands AB, ed. Marmosets and Tamarins: Systematics, Behaviour, and Ecology. Oxford University Press; 1993:262-272.

29. Kap YS, Bus-Spoor C, van Driel N, et al. Targeted Diet Modification Reduces Multiple Sclerosis-like Disease in Adult Marmoset Monkeys from an Outbred Colony. J Immunol. 2018;201(11):3229-3243. doi:10.4049/jimmunol.1800822

30. Ross CN, Austad S, Brasky K, et al. The development of a specific pathogen free (SPF) barrier colony of marmosets (Callithrix jacchus) for aging research. Aging (Albany NY). 2017;9(12):2544-2558. doi:10.18632/aging.101340

31. Reveles KR, Patel S, Forney L, Ross CN. Age-related changes in the marmoset gut microbiome. Am J Primatol. 2019;81(2). doi:10.1002/ajp.22960

32. Artim SC, Sheh A, Burns MA, Fox JG. Evaluating rectal swab collection method for gut microbiome analysis in the common marmoset (Callithrix jacchus). PLoS One.

2019;14(11). doi:10.1371/journal.pone.0224950

33. Kobayashi R, Nagaoka K, Nishimura N, et al. Comparison of the fecal microbiota of two monogastric herbivorous and five omnivorous mammals. Anim Sci J. 2020;91(1):e13366. doi:10.1111/asj.13366

34. Zhu L, Clayton JB, Suhr Van Haute MJ, et al. Sex Bias in Gut Microbiome Transmission in Newly Paired Marmosets ( Callithrix jacchus ). mSystems. 2020;5(2). doi:10.1128/msystems.00910-19

35. Uzal FA, Navarro MA, Li J, Freedman JC, Shrestha A, McClane BA. Comparative pathogenesis of enteric clostridial infections in humans and animals. Anaerobe. 2018;53:11-20. doi:10.1016/j.anaerobe.2018.06.002

36. Mazuet C, Legeay C, Sautereau J, et al. Characterization of Clostridium Baratii Type F Strains Responsible for an Outbreak of Botulism Linked to Beef Meat Consumption in France. PLoS Curr. 2017;9. doi:10.1371/currents.outbreaks.6ed2fe754b58a5c42d0c33d586ffc606

37. Duvallet C, Gibbons SM, Gurry T, Irizarry RA, Alm EJ. Meta-analysis of gut microbiome studies identifies disease-specific and shared responses. Nat Commun. 2017;8(1):1-10. doi:10.1038/s41467-017-01973-8

38. Arumugam M, Raes J, Pelletier E, et al. Enterotypes of the human gut microbiome. Nature. 2011;473(7346):174-180. doi:10.1038/nature09944

39. Potkay S. Diseases of the Callitrichidae: a review. J Med Primatol. 1992;21(4):189-236. Accessed July 13, 2020. https://europepmc.org/article/med/1527793

40. McKenzie VJ, Jin Song S, Delsuc F, et al. The Effects of Captivity on the Mammalian Gut Microbiome Society for Integrative and Comparative Biology. Integr Comp Biol. 2017;57(4):690-704. doi:10.1093/icb/icx090 
704

705

706

707

708

709

710

711

712

713

714

715

716

717

718

719

720

721

722

723

724

725

726

727

728

729

730

731

732

733

734

41. Kovatcheva-Datchary P, Nilsson A, Akrami R, et al. Dietary Fiber-Induced Improvement in Glucose Metabolism Is Associated with Increased Abundance of Prevotella. Cell Metab. 2015;22(6):971-982. doi:10.1016/j.cmet.2015.10.001

42. David LA, Maurice CF, Carmody RN, et al. Diet rapidly and reproducibly alters the human gut microbiome. Nature. 2014;505(7484):559-563. doi:10.1038/nature12820

43. Louis P, Hold GL, Flint HJ. The gut microbiota, bacterial metabolites and colorectal cancer. Nat Rev Microbiol. 2014;12(10):661-672. doi:10.1038/nrmicro3344

44. Tsukahara T, Koyama H, Okada M, Ushida K. Stimulation of butyrate production by gluconic acid in batch culture of pig cecal digesta and identification of butyrate-producing bacteria - PubMed. J Nutr. 2002;132(8):2229-2234. Accessed July 13, 2020. https://pubmed.ncbi.nlm.nih.gov/12163667/

45. Albert K, Rani A, Sela DA. The comparative genomics of Bifidobacterium callitrichos reflects dietary carbohydrate utilization within the common marmoset gut. Microb genomics. 2018;4(6). doi:10.1099/mgen.0.000183

46. Cooper RE, Mangus L, Wright J, Lamendella R, Mankowski J. Abstracts of Scientific Presentations 2019 AALAS National Meeting: PS59 Gut Microbiota Alterations in Marmoset Wasting Syndrome: A Cross-Population Study. J Am Assoc Lab Anim Sci. 2019;58(5):607-726. Accessed July 13, 2020. https://www.ncbi.nlm.nih.gov/pmc/articles/PMC6774462/

47. Pinheiro HLN, Mendes Pontes AR. Home range, diet, and activity patterns of common marmosets (Callithrix Jacchus) in very small and isolated fragments of the Atlantic forest of northeastern Brazil. Int J Ecol. 2015;2015. doi:10.1155/2015/685816

48. Clayton JB, Vangay P, Huang H, et al. Captivity humanizes the primate microbiome. Proc Natl Acad Sci U S A. 2016;113(37):10376-10381. doi:10.1073/pnas.1521835113

49. Malnick H, Williams K, Phil-Ebosie J, Levy AS. Description of a medium for isolating Anaerobiospirillum spp., a possible cause of zoonotic disease, from diarrheal feces and blood of humans and use of the medium in a survey of human, canine, and feline feces. $J$ Clin Microbiol. 1990;28(6):1380-1384. doi:10.1128/jcm.28.6.1380-1384.1990

50. Yasuda $\mathrm{M}$, Inoue $\mathrm{T}$, Ueno $\mathrm{M}$, et al. A case of nontraumatic gas gangrene in a common marmoset (Callithrix jacchus). J Vet Med Sci. 2016;77(12):1673-1676. doi:10.1292/jvms. 15-0210

51. Christie RJ, King RE. Acute gastric dilatation and rupture in Macaca arctoides associated with Clostridium perfringens. J Med Primatol. 1981;10(4-5):263-264. doi:10.1159/000460083

52. Meier TR, Myers, Daniel D, Eaton KA, Ko MH, Hankenson FC. Gangrenous Clostridium perfringens Infection and Subsequent Wound Management in a Rhesus Macaque (Macaca mulatta). J Am Assoc Lab Anim Sci. 2007;46(4):68-73.

53. Holland D, Thomson L, Mahmoudzadeh N, Khaled A. Estimating deaths from foodborne disease in the UK for 11 key pathogens. BMJ Open Gastroenterol. 2020;7(1):e000377. doi:10.1136/bmjgast-2020-000377 
54. De La Cochetière MF, Piloquet H, Des Robert C, Darmaun D, Galmiche JP, Rozé JC. Early intestinal bacterial colonization and necrotizing enterocolitis in premature infants: The putative role of Clostridium. Pediatr Res. 2004;56(3):366-370. doi:10.1203/01.PDR.0000134251.45878.D5

55. Janik JS, Ein SH, Mancer K. Intestinal stricture after necrotizing enterocolitis. J Pediatr Surg. 1981;16(4):438-443. doi:10.1016/S0022-3468(81)80002-4

56. Phad N, Trivedi A, Todd D, Lakkundi A. Intestinal strictures post-necrotising enterocolitis: clinical profile and risk factors. J neonatal Surg. 2014;3(4):44. doi:10.21699/jns.v3i4.184

57. Neu J, Pammi M. Necrotizing enterocolitis: The intestinal microbiome, metabolome and inflammatory mediators. Semin Fetal Neonatal Med. 2018;23(6):400-405. doi:10.1016/j.siny.2018.08.001

58. Ridlon JM, Kang DJ, Hylemon PB. Bile salt biotransformations by human intestinal bacteria. J Lipid Res. 2006;47(2):241-259. doi:10.1194/jlr.R500013-JLR200

59. Wang S, Martins R, Sullivan MC, et al. Diet-induced remission in chronic enteropathy is associated with altered microbial community structure and synthesis of secondary bile acids. Microbiome. 2019;7(1):1-20. doi:10.1186/s40168-019-0740-4

60. Patel RM, Knezevic A, Shenvi N, et al. Association of red blood cell transfusion, anemia, and necrotizing enterocolitis in very low-birth-weight infants. JAMA - J Am Med Assoc. 2016;315(9):889-897. doi:10.1001/jama.2016.1204

61. Manni M, Valero JG. Lipidomic profile of GM95 cell death induced by Clostridium perfringens alpha- toxin. 2017;(January). doi:10.1016/j.chemphyslip.2017.01.002

62. Joossens M, Huys G, Cnockaert M, et al. Dysbiosis of the faecal microbiota in patients with Crohn's disease and their unaffected relatives. Gut. 2011;60(5):631-637. doi:10.1136/gut.2010.223263

63. Paramsothy S, Nielsen S, Kamm MA, et al. Specific Bacteria and Metabolites Associated With Response to Fecal Microbiota Transplantation in Patients With Ulcerative Colitis. Gastroenterology. 2019;156(5):1440-1454.e2. doi:10.1053/j.gastro.2018.12.001

64. Hyams JS, Davis Thomas S, Gotman N, et al. Clinical and biological predictors of response to standardised paediatric colitis therapy (PROTECT): a multicentre inception cohort study. Lancet. 2019;393(10182):1708-1720. doi:10.1016/S0140-6736(18)32592-3

65. Moon C, Baldridge MT, Wallace MA, Burnham CAD, Virgin HW, Stappenbeck TS. Vertically transmitted faecal IgA levels determine extra-chromosomal phenotypic variation. Nature. 2015;521(7550):90-93. doi:10.1038/nature14139

66. Connors J, Dunn KA, Allott J, et al. The relationship between fecal bile acids and microbiome community structure in pediatric Crohn's disease. ISME J. 2020;14(3):702713. doi:10.1038/s41396-019-0560-3

67. Hehemann JH, Correc G, Barbeyron T, Helbert W, Czjzek M, Michel G. Transfer of carbohydrate-active enzymes from marine bacteria to Japanese gut microbiota. Nature. 
775

776

777

778

779

780

781

782

783

784

785

786

787

788

789

790

791

792

793

794

795

796

797

798

799

800

801

802

803

804

805

806

807

808

809

810

811

68. Wexler HM. Bacteroides: The good, the bad, and the nitty-gritty. Clin Microbiol Rev. 2007;20(4):593-621. doi:10.1128/CMR.00008-07

69. Delday M, Mulder I, Logan E, Grant G. Bacteroides thetaiotaomicron Ameliorates Colon Inflammation in Preclinical Models of Crohn's Disease. Inflamm Bowel Dis. 2019;25(1). doi:10.1093/IBD/IZY281

70. Bloom SM, Bijanki VN, Nava GM, et al. Commensal Bacteroides species induce colitis in host-genotype-specific fashion in a mouse model of inflammatory bowel disease. Cell Host Microbe. 2011;9(5):390-403. doi:10.1016/j.chom.2011.04.009

71. Lucke K, Miehlke S, Jacobs E, Schuppler M. Prevalence of Bacteroides and Prevotella spp. in ulcerative colitis. J Med Microbiol. 2006;55(5):617-624.

doi:10.1099/jmm.0.46198-0

72. Swidsinski A, Ladhoff A, Pernthaler A, et al. Mucosal flora in inflammatory bowel disease. Gastroenterology. 2002;122(1):44-54. doi:10.1053/gast.2002.30294

73. Larsen JM. The immune response to Prevotella bacteria in chronic inflammatory disease. Immunology. 2017;151(4):363-374. doi:10.1111/imm.12760

74. Scher JU, Sczesnak A, Longman RS, et al. Expansion of intestinal Prevotella copri correlates with enhanced susceptibility to arthritis. Elife. 2013;2013(2). doi:10.7554/eLife.01202.001

75. Pianta A, Arvikar S, Strle K, et al. Evidence of the Immune Relevance of Prevotella copri, a Gut Microbe, in Patients With Rheumatoid Arthritis. Arthritis Rheumatol. 2017;69(5):964-975. doi:10.1002/art.40003

76. de Aquino SG, Abdollahi-Roodsaz S, Koenders MI, et al. Periodontal Pathogens Directly Promote Autoimmune Experimental Arthritis by Inducing a TLR2- and IL-1-Driven Th17 Response. J Immunol. 2014;192(9):4103-4111. doi:10.4049/jimmunol.1301970

77. Maeda Y, Kurakawa T, Umemoto E, et al. Dysbiosis Contributes to Arthritis Development via Activation of Autoreactive T Cells in the Intestine. Arthritis Rheumatol. 2016;68(11):2646-2661. doi:10.1002/art.39783

78. Elinav E, Strowig T, Kau AL, et al. NLRP6 inflammasome regulates colonic microbial ecology and risk for colitis. Cell. 2011;145(5):745-757. doi:10.1016/j.cell.2011.04.022

79. $\mathrm{Su}$ T, Liu R, Lee A, et al. Altered Intestinal Microbiota With Increased Abundance of Prevotella Is Associated With High Risk of Diarrhea-Predominant Irritable Bowel Syndrome. Gastroenterol Res Pract. 2018;2018. doi:10.1155/2018/6961783

80. Vázquez-Castellanos JF, Serrano-Villar S, Latorre A, et al. Altered metabolism of gut microbiota contributes to chronic immune activation in HIV-infected individuals. Mucosal Immunol. 2015;8(4):760-772. doi:10.1038/mi.2014.107

81. Rey J, Giustiniani J, Mallet F, et al. The co-expression of 2B4 (CD244) and CD160 delineates an subpopulation of human CD8+ T cells with a potent CD160-mediated 
cytolytic effector function. Eur J Immunol. 2006;36(9):2359-2366. doi:10.1002/eji.200635935

82. Bloch-Queyrat $\mathrm{C}$, Fondanèche $\mathrm{MC}$, Chen $\mathrm{R}$, et al. Regulation of natural cytotoxicity by the adaptor SAP and the Src-related kinase Fyn. J Exp Med. 2005;202(1):181-192. doi:10.1084/jem.20050449

83. Poggi A, Benelli R, Venè R, et al. Human gut-associated natural killer cells in health and disease. Front Immunol. 2019;10(MAY):961. doi:10.3389/fimmu.2019.00961

84. Fox JG, Dangler CA, Taylor NS, King A, Koh TJ, Wang TC. High-Salt Diet Induces Gastric Epithelial Hyperplasia and Parietal Cell Loss, and Enhances Helicobacter pylori Colonization in C57BL/6 Mice | Cancer Research. Cancer Res. 1999;59(19):4823-4828.

85. Comeau AM, Douglas GM, Langille MGI. Microbiome Helper: a Custom and Streamlined Workflow for Microbiome Research. mSystems. 2017;2(1):e00127-16. doi:10.1128/mSystems.00127-16

86. Andrews S. FastQC A Quality Control tool for High Throughput Sequence Data. Published 2010. Accessed August 4, 2020. http:/www.bioinformatics.babraham.ac.uk/projects/fastqc/

87. Bolyen E, Rideout JR, Dillon MR, et al. Reproducible, interactive, scalable and extensible microbiome data science using QIIME 2. Nat Biotechnol. 2019;37(8):852-857. doi:10.1038/s41587-019-0209-9

88. Martin M. Cutadapt removes adapter sequences from high-throughput sequencing reads. EMBnet.journal. 2011;17(1):10. doi:10.14806/ej.17.1.200

89. Yilmaz P, Wegener Parfrey L, Yarza P, et al. SILVA and “All-species Living Tree Project (LTP)" taxonomic frameworks | Nucleic Acids Research | Oxford Academic. Nucleic Acids Res. 2014;42(D1):D643-D648.

90. Mandal S, Van Treuren W, White RA, Eggesbø M, Knight R, Peddada SD. Analysis of composition of microbiomes: a novel method for studying microbial composition. Microb Ecol Heal Dis. 2015;26(0). doi:10.3402/mehd.v26.27663

91. Lozupone C, Hamady M, Knight R. UniFrac--an online tool for comparing microbial community diversity in a phylogenetic context. BMC Bioinformatics. 2006;7(1):371. doi:10.1186/1471-2105-7-371

92. Wickham H. ggplot2: elegant graphics for data analysis. Springer;. Published online 2009.

93. Kuhn M. Building predictive models in R using the caret package. J Stat Softw. 2008;28(5):1-26. doi:10.18637/jss.v028.i05

94. Oksanen J, Kindt R, Legendre P, et al. The vegan Package. Published online 2008. Accessed August 4, 2020. http://cran.r-project.org/,

95. Robin X, Turck N, Hainard A, et al. pROC: An open-source package for R and S+ to analyze and compare ROC curves. BMC Bioinformatics. 2011;12(1):77.

doi:10.1186/1471-2105-12-77 
96. Warns Gregory, Bolker Ben LT. gtools: Various R Programming Tools. Published online 2015.

97. Lee CM, Barber GP, Casper J, et al. UCSC Genome Browser enters 20th year. Nucleic Acids Res. 2020;48. doi:10.1093/nar/gkz1012

98. Liao Y, Smyth GK, Shi W. The R package Rsubread is easier, faster, cheaper and better for alignment and quantification of RNA sequencing reads. Nucleic Acids Res. 2019;47(8). doi:10.1093/nar/gkz114

99. Robinson MD, Mccarthy DJ, Smyth GK. edgeR: a Bioconductor package for differential expression analysis of digital gene expression data. Bioinforma Appl NOTE. 2010;26(1):139-140. doi:10.1093/bioinformatics/btp616

100. Mccarthy DJ, Chen Y, Smyth GK. Differential expression analysis of multifactor RNASeq experiments with respect to biological variation. Nucleic Acids Res. 2012;40:42884297. doi:10.1093/nar/gks042

101. Ritchie ME, Phipson B, Wu D, et al. limma powers differential expression analyses for RNA-sequencing and microarray studies. Nucleic Acids Res. 2015;43(7). doi:10.1093/nar/gkv007

102. Pagès H, Carlson M, Falcon S, Li N. AnnotationDbi: Manipulation of SQLite-based annotations in Bioconductor. R package. Published online 2019.

103. Carlson M. GO.db: A set of annotation maps describing the entire Gene Ontology. R package. Published online 2019.

104. Alexa A, Rahnenfuhrer J. topGO: Enrichment Analysis for Gene Ontology. R package. Published online 2019.

105. Mark A, Thompson R, Afrasiabi C, Wu C. mygene: Access MyGene.Info_services. R package version. Published online 2019.

106. Hansen KD, Gentry J, Long L, et al. Rgraphviz: Provides plotting capabilities for R graph objects. R package. Published online 2019.

107. Zeileis A, Fisher JC, Hornik K, et al. colorspace: A Toolbox for Manipulating and Assessing Colors and Palettes. Published online March 14, 2019. Accessed August 4, 2020. http://arxiv.org/abs/1903.06490

108. Gao C-H. ggVennDiagram: A “ggplot” Implement of Venn Diagram. R package. Published online 2019. https://cran.r-project.org/package=ggVennDiagram 


\section{Figure 1}

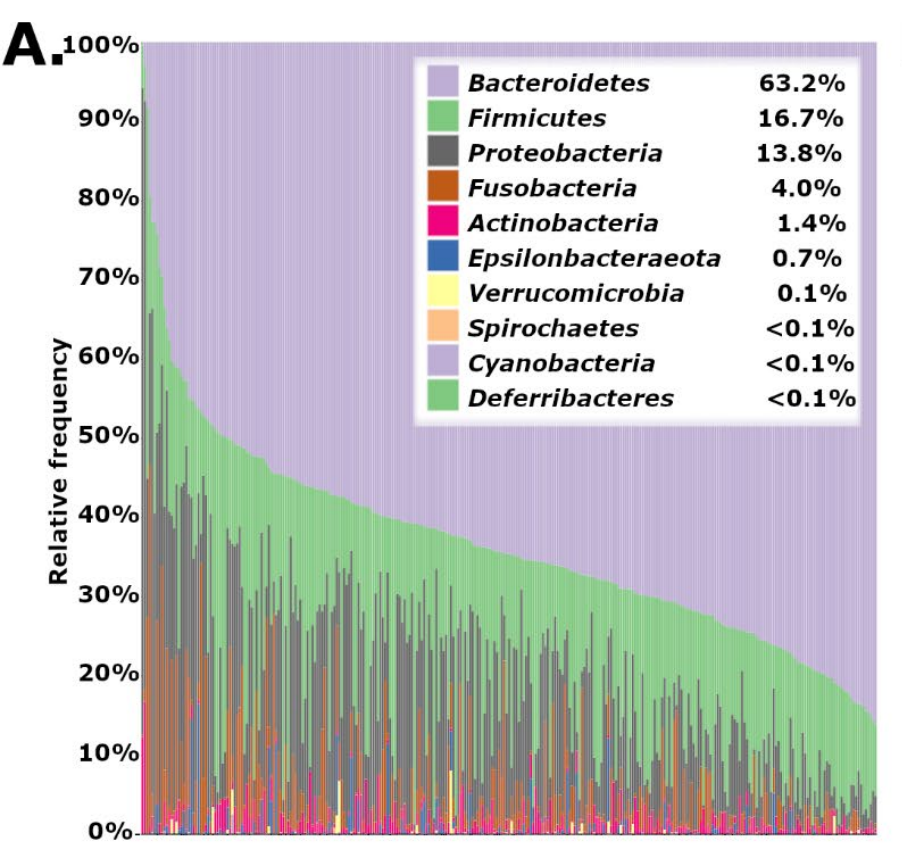

C.

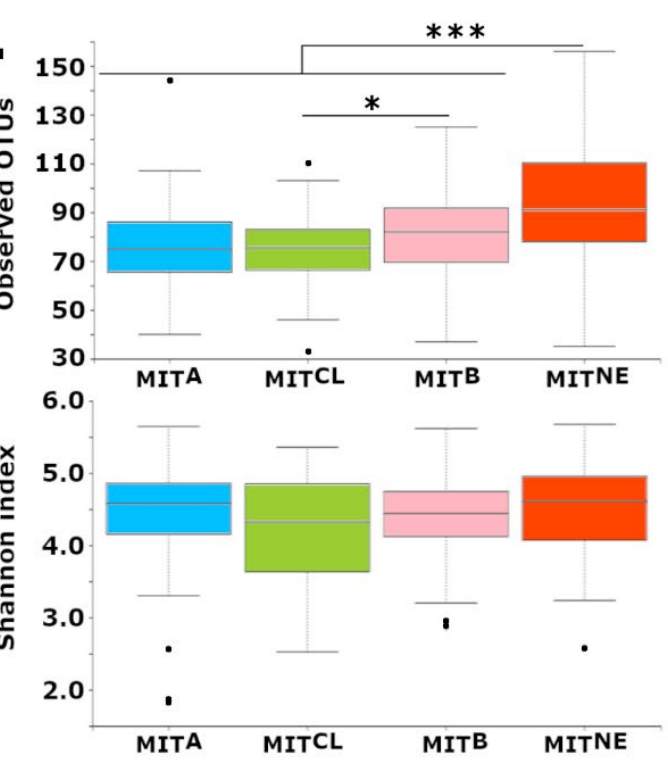

B.

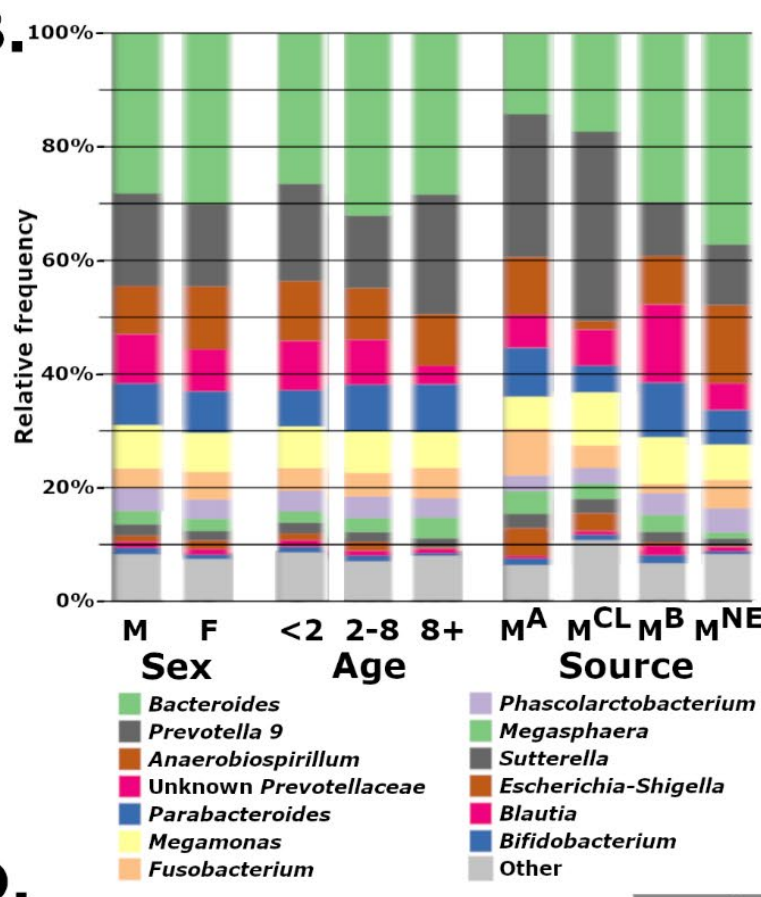

D. Fusobacterium

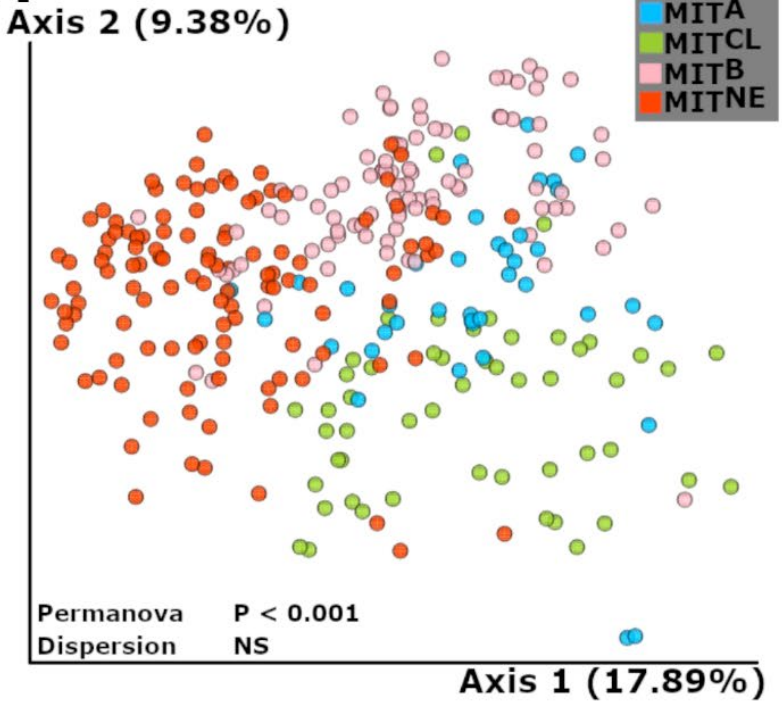

Figure 1. A) Gut microbiome profiles of healthy, common marmosets at the phylum level exhibit a Bacteroidetes-dominant and human-like microbiome. B) Averaged relative abundances at the genus level show differences associated with source but few differences based on sex or age. C) Observed OTUs were increased in $\mathrm{MIT}^{\mathrm{NE}}$ vs. all sources and $\mathrm{MIT}^{\mathrm{B}}$ compared to $\mathrm{MIT}^{\mathrm{A}}$ and $\mathrm{MIT} \mathrm{TL}^{\mathrm{CL}}$, but metrics involving evenness, such as Shannon's diversity index, showed no difference. ${ }^{*}, P<0.05 ; * *, P<0.01$ and $* * *, P<0.001$. D) PCoA plot using Unweighted UniFrac metric shows clustering of microbiome profiles based on marmoset source. 


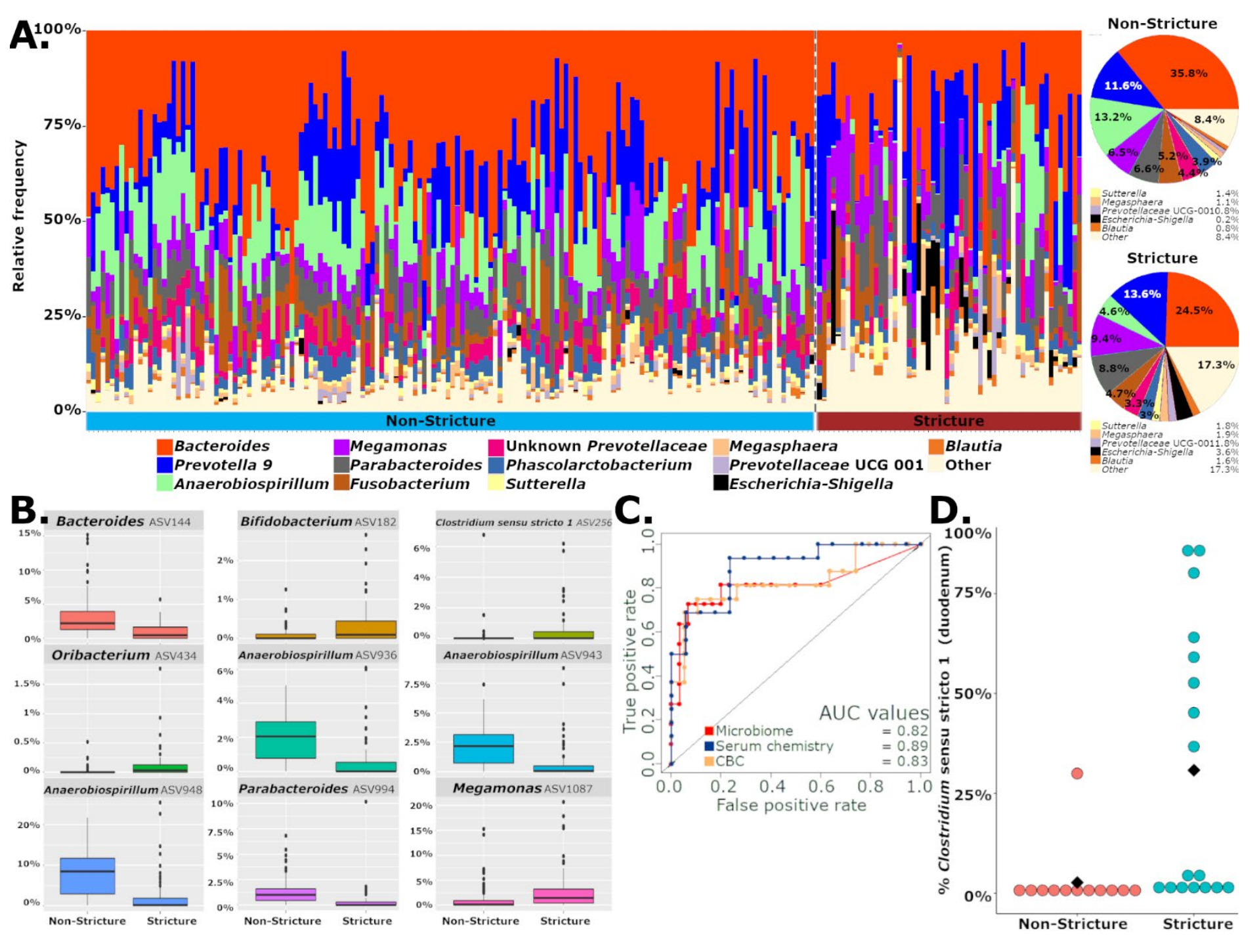

Figure 2. A) Microbiome composition of samples at the genus level and pie charts with average bacterial abundances of stricture progressors and nonprogressors show dysbiosis associated with stricture characterized by decreased Bacteroides and Anaerobiospirillum and increased Megamonas. B) Nine ASVs identified by a random forest model that can correctly classify stricture and non-stricture samples with a $85 \%$ accuracy. C) Area under the curve (AUC) of receiver operating characteristic (ROC) curves for random forest models using microbiome, serum chemistry or complete blood count show strong performance of models in classifying strictures and non-strictures. D) Relative abundance of Clostridium sensu stricto 1 reads in duodenal biopsies is increased in stricture cases compared to non-stricture cases. 


\section{Figure 3}

$\mathbf{A}_{18}$

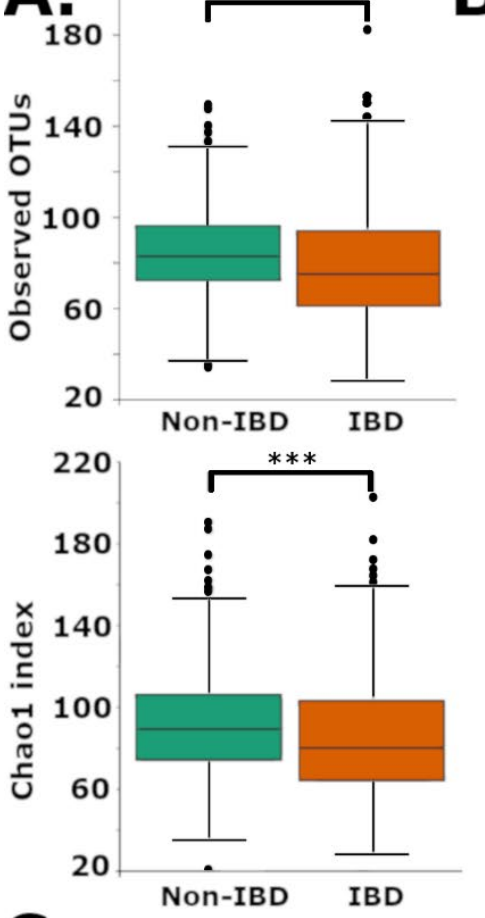

B.

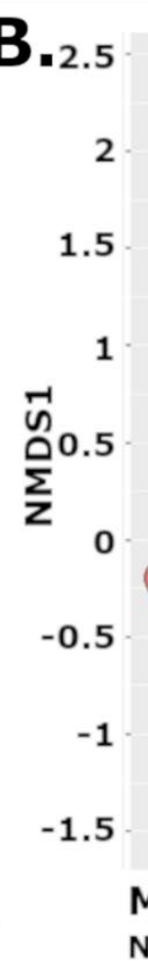

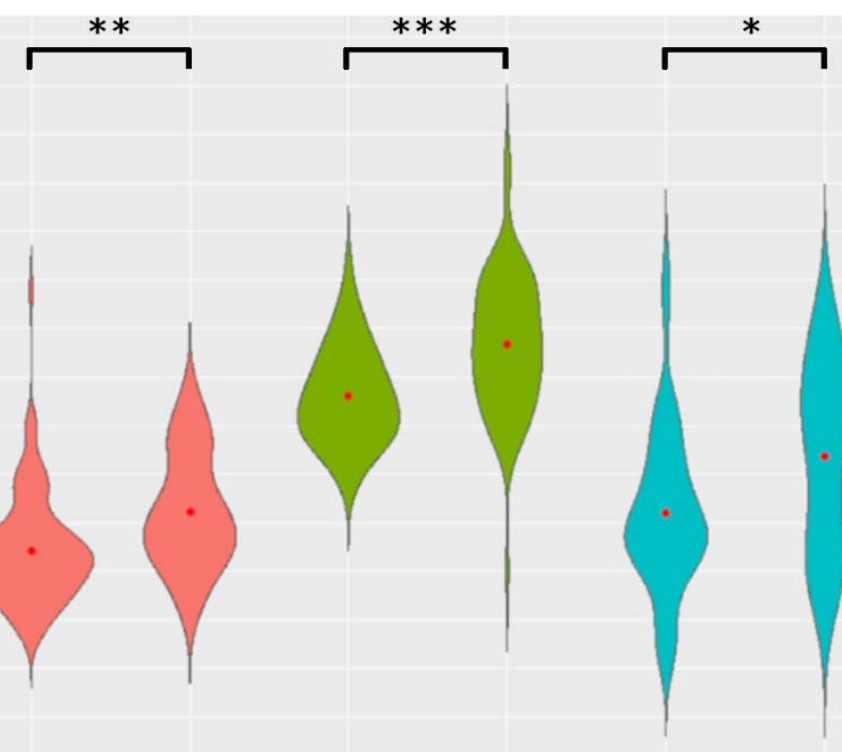

Figure 3. A) Decreased richness was observed in IBD marmosets (Observed OTUs and Chao1) compared to non-IBD marmosets similar to what is observed in humans. B) Increases in $\mathrm{PC1}$ relative to sourcespecific, non-IBD controls were observed in 3 of 4 sources. C) Bacteroides and Prevotella 9 levels are shown by source and IBD status. A lower overall and source-specific Bacteroides:Prevotella 9 ratio is MIT'B MIT'B MITCL MITCL MITA MITA MITNE MITNE observed in IBD cases regardless of source-specific Non-IBD IBD Non-IBD IBD Non-IBD IBD Non-IBD IBD
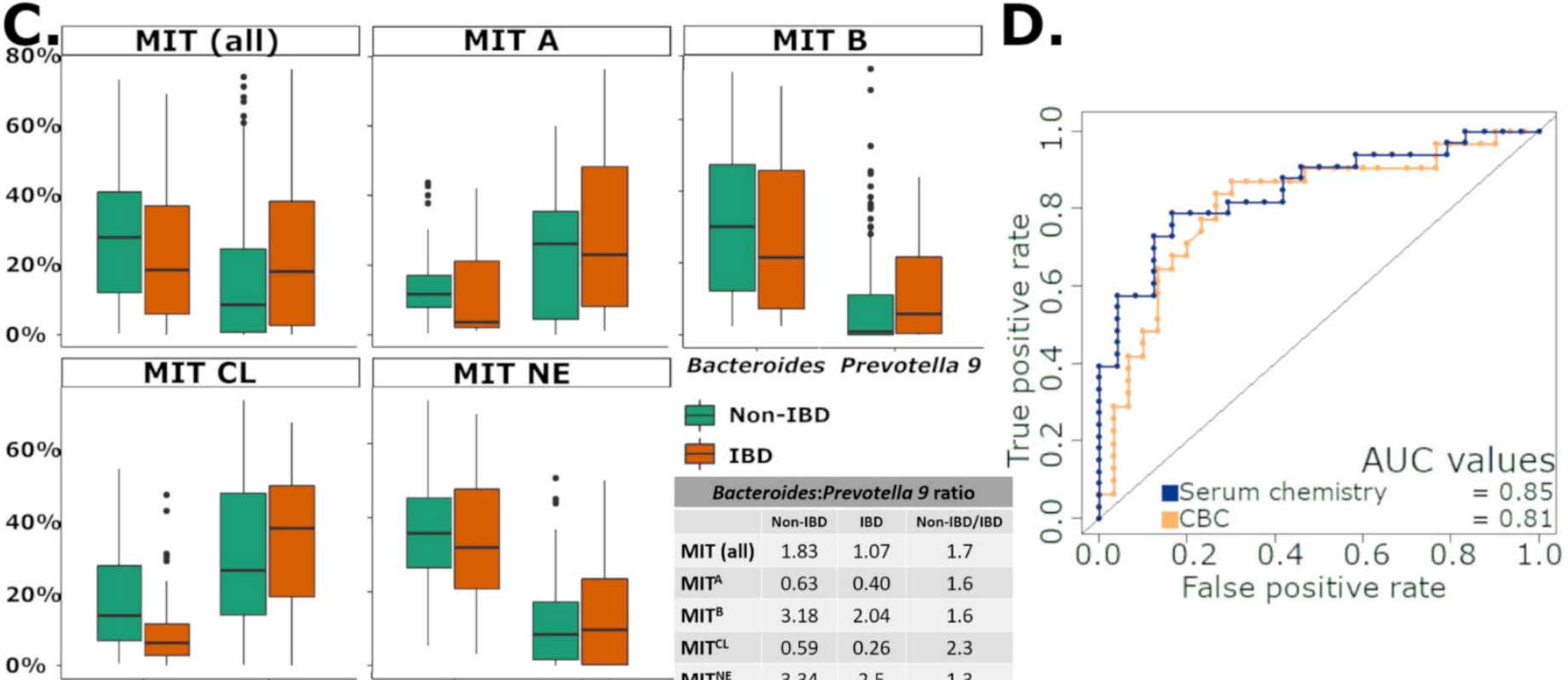
differences in abundances of these two genera. D) AUC of ROC for random forest models using serum chemistry and $\mathrm{CBC}$ show strong performance of models in classifying IBD progressors and nonprogressors. *, $P<0.05 ; * *, \quad P<0.01$ and ***, $P<0.001$. 
A.

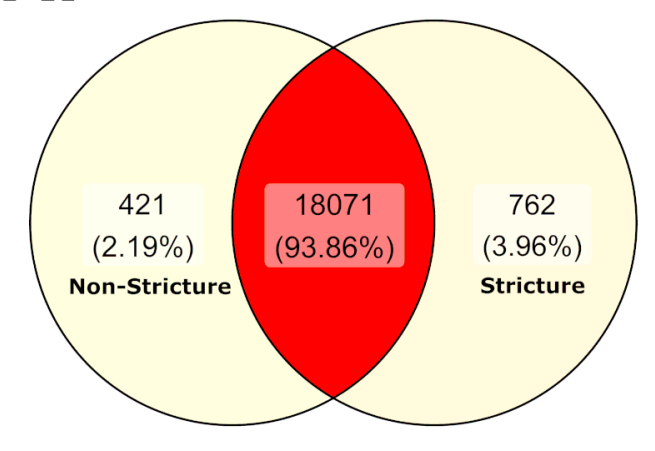

C.

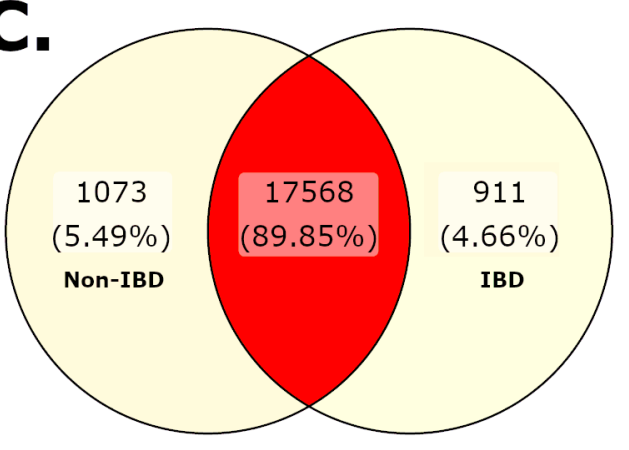

B.

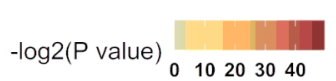

cellular response to
cytokine stimulus cytokine stimulus
response to stimulus

intestinal absorption-

organelle fission-
glycerolipid

glycerolipid
metabolic process
small molecule

small molecule
metabolic process
cellutar Tipid

metabolic process

proliferation
carboxylic acid
metabolic process

metabolic process
antimicrobial
humoral response

organic anion transport-

lipid localization-

regulation of-
hormone levels

cell proliferation-

immune response-

defense response

response to stress

lipid transport-

anion transport-

ion transport-

acylglycerol
metabolic process
nic

metabolic process

lipid metabolic process

metabolic process
leukocyte

immune system process

Immune system process
leukocyte activation involved

in immune response
cell activation involved in
immune response

immune effector process

nuclear division-

Non-Stricture
D.

-log2(P value) 010203040

trans-synaptic
signaling

synaptic signaling

anterograde trans-
synaptic signaling

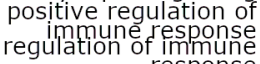

regulation of response
anatomical structure

system development

cell development

lymphocyte activation

leukocyte activation

regulation of system
process

$\mathrm{T}$ cell activation
developmental

multiceless
rerganismal process

behavior

neryous system
development

chemical synaptic
transmission

leukocyte cell-cell
adhesion

immune response

defense response

muscle contraction

muscle system
process

system process

positive regulation of
immune systeml arocess
regulation of immune

ecrocye differentiation

immune system procen

immun system process

mune effector process
adaptive immune
response

cell activation

Non-IBD
Figure 4. A) Differentially expressed genes $(\mathrm{DEG})(\mathrm{FDR}<0.05)$ in the duodenum of nonstricture and stricture cases. B) Gene ontology (GO) sets enriched in stricture cases show upregulation of lipid metabolism, transport and localization. Non-stricture cases have enrichment of immune processes, possibly due to underlying pathology caused by IBD. C) DEG (FDR $<0.05)$ in the jejunum of non-IBD and IBD cases. D) IBD samples are enrich GO sets associated with immunity and immune cell activation. 


\begin{tabular}{|c|c|c|c|c|}
\hline & & Healthy & Stricture & IBD \\
\hline \multirow{2}{*}{ Sex } & Male & 156 & 25 & 95 \\
\hline & Female & 147 & 37 & 105 \\
\hline \multirow{3}{*}{ Age } & 2 and under & 145 & 19 & 36 \\
\hline & 2 to 8 & 139 & 42 & 131 \\
\hline & Over 8 & 19 & 1 & 33 \\
\hline \multirow{2}{*}{ Type } & Rectal & 207 & 24 & 111 \\
\hline & Fecal & 96 & 38 & 89 \\
\hline \multirow{4}{*}{ Source } & $\mathrm{MIT}^{\mathrm{NE}}$ & 117 & 57 & 89 \\
\hline & $\mathrm{MIT}^{\mathrm{B}}$ & 94 & 3 & 30 \\
\hline & $\mathrm{MIT}^{\mathrm{CL}}$ & 53 & 2 & 61 \\
\hline & $\mathrm{MIT}^{\mathrm{A}}$ & 39 & 0 & 20 \\
\hline
\end{tabular}


Table 2. Top Gene Ontology sets in Stricture

Biological Processes upregulated in the Duodenum in Stricture

\begin{tabular}{|c|c|c|c|c|c|c|c|}
\hline GO ID & Term & Ont & $\mathbf{N}$ & Up & Down & P.Up & P.Down \\
\hline GO:0010876 & lipid localization & $\mathrm{BP}$ & 292 & 14 & 44 & $1.08 \mathrm{E}-02$ & $1.39 \mathrm{E}-12$ \\
\hline GO:0006629 & lipid metabolic process & $\mathrm{BP}$ & 1097 & 27 & 99 & $4.69 \mathrm{E}-01$ & $1.02 \mathrm{E}-11$ \\
\hline GO:0006869 & lipid transport & $\mathrm{BP}$ & 262 & 13 & 39 & $1.05 \mathrm{E}-02$ & $3.98 \mathrm{E}-11$ \\
\hline GO:0046486 & glycerolipid metabolic process & $\mathrm{BP}$ & 349 & 10 & 45 & $3.26 \mathrm{E}-01$ & $1.70 \mathrm{E}-10$ \\
\hline GO:0044281 & small molecule metabolic process & $\mathrm{BP}$ & 1646 & 49 & 128 & $6.11 \mathrm{E}-02$ & $1.80 \mathrm{E}-10$ \\
\hline GO:0044255 & cellular lipid metabolic process & $\mathrm{BP}$ & 842 & 22 & 79 & $3.65 \mathrm{E}-01$ & $2.81 \mathrm{E}-10$ \\
\hline GO:0006639 & acylglycerol metabolic process & $\mathrm{BP}$ & 104 & 4 & 22 & $2.38 \mathrm{E}-01$ & $1.00 \mathrm{E}-09$ \\
\hline GO:0006638 & neutral lipid metabolic process & $\mathrm{BP}$ & 105 & 4 & 22 & 0.24339141 & $1.22 \mathrm{E}-09$ \\
\hline GO:0015711 & organic anion transport & $\mathrm{BP}$ & 364 & 16 & 44 & 0.01443175 & $2.16 \mathrm{E}-09$ \\
\hline GO:0006811 & ion transport & $\mathrm{BP}$ & 1196 & 39 & 98 & 0.02879741 & 2.79E-09 \\
\hline GO:0006820 & anion transport & $\mathrm{BP}$ & 459 & 19 & 50 & 0.01459 & $6.00 \mathrm{E}-09$ \\
\hline GO:0006082 & organic acid metabolic process & $\mathrm{BP}$ & 923 & 27 & 80 & 0.16158189 & $8.66 \mathrm{E}-09$ \\
\hline GO:0019752 & carboxylic acid metabolic process & $\mathrm{BP}$ & 845 & 27 & 75 & 0.07620236 & $9.78 \mathrm{E}-09$ \\
\hline GO:0010817 & regulation of hormone levels & $\mathrm{BP}$ & 377 & 10 & 43 & 0.41543649 & $1.90 \mathrm{E}-08$ \\
\hline GO:0050892 & intestinal absorption & $\mathrm{BP}$ & 35 & 0 & 12 & 1 & $2.04 \mathrm{E}-08$ \\
\hline
\end{tabular}

Biological processes upregulated in the Duodenum in Non-Stricture

\section{GO ID}

GO:0002376

GO:0006955

GO:0019730

GO:0006950

GO:0008283

GO:0000280

GO:0002252

GO:0006952

GO:0002443

GO:0048285

GO:0050896

GO:0042127

GO:0002366

GO:0002263

GO:0071345

\section{Term}

immune response

antimicrobial humoral response

response to stress

cell proliferation

nuclear division

immune effector process

defense response

leukocyte mediated immunity

organelle fission

response to stimulus

regulation of cell proliferation

leukocyte activation involved in immune response

cell activation involved in immune response

cellular response to cytokine stimulus

\begin{tabular}{cccccc} 
Ont & $\mathbf{N}$ & Up & Down & P.Up & P.Down \\
\hline BP & 2192 & 110 & 86 & $1.24 \mathrm{E}-15$ & $9.36 \mathrm{E}-01$ \\
BP & 1482 & 83 & 50 & $4.21 \mathrm{E}-14$ & $9.91 \mathrm{E}-01$ \\
BP & 49 & 13 & 0 & $7.97 \mathrm{E}-11$ & $1.00 \mathrm{E}+00$ \\
BP & 3001 & 122 & 106 & $9.11 \mathrm{E}-11$ & $9.99 \mathrm{E}-01$ \\
BP & 1479 & 75 & 56 & $1.33 \mathrm{E}-10$ & $9.35 \mathrm{E}-01$ \\
BP & 322 & 30 & 7 & $1.81 \mathrm{E}-10$ & $9.92 \mathrm{E}-01$ \\
BP & 900 & 54 & 31 & $2.54 \mathrm{E}-10$ & $9.58 \mathrm{E}-01$ \\
BP & 1138 & 62 & 38 & $4.85 \mathrm{E}-10$ & $9.84 \mathrm{E}-01$ \\
BP & 598 & 41 & 21 & $1.05 \mathrm{E}-09$ & $9.08 \mathrm{E}-01$ \\
BP & 357 & 30 & 8 & $2.16 \mathrm{E}-09$ & $9.92 \mathrm{E}-01$ \\
BP & 6351 & 203 & 305 & $3.03 \mathrm{E}-09$ & $6.44 \mathrm{E}-02$ \\
BP & 1226 & 63 & 47 & $3.54 \mathrm{E}-09$ & $9.01 \mathrm{E}-01$ \\
BP & 559 & 38 & 21 & $5.77 \mathrm{E}-09$ & 0.83699294 \\
BP & 562 & 38 & 21 & $6.68 \mathrm{E}-09$ & 0.84361261 \\
BP & 849 & 49 & 31 & $7.05 \mathrm{E}-09$ & 0.91205517
\end{tabular}


Table 3. Top Gene Ontology sets in IBD

Biological processes upregulated in the Jejunum in IBD

GO ID Term

GO:0002376 immune system process

GO:0006955 immune response

GO:0045321 leukocyte activation

GO:0046649 lymphocyte activation

GO:0042110 T cell activation

GO:0002682 regulation of immune system process

GO:0001775 cell activation

GO:0002250 adaptive immune response

GO:0002684 positive regulation of immune system process

GO:0050776 regulation of immune response

GO:0006952 defense response

GO:0002252 immune effector process

GO:0050778 positive regulation of immune response

GO:0002521 leukocyte differentiation

GO:0007159 leukocyte cell-cell adhesion

$\begin{array}{cccccc}\text { Ont } & \mathbf{N} & \text { Up } & \text { Down } & \text { P.Up } & \text { P.Down } \\ \text { BP } & 2197 & 90 & 286 & 1 & 7.30 \mathrm{E}-58 \\ \text { BP } & 1473 & 39 & 227 & 1 & 7.75 \mathrm{E}-57 \\ \text { BP } & 949 & 24 & 165 & 1 & 7.62 \mathrm{E}-47 \\ \text { BP } & 516 & 11 & 119 & 0.9999998 & 4.13 \mathrm{E}-46 \\ \text { BP } & 361 & 7 & 97 & 0.9999956 & 1.32 \mathrm{E}-43 \\ \text { BP } & 1154 & 40 & 177 & 0.9999999 & 1.42 \mathrm{E}-42 \\ \text { BP } & 1071 & 37 & 169 & 0.9999997 & 3.75 \mathrm{E}-42 \\ \text { BP } & 282 & 3 & 81 & 0.9999995 & 9.97 \mathrm{E}-39 \\ \text { BP } & 816 & 20 & 136 & 1 & 5.02 \mathrm{E}-36 \\ \text { BP } & 758 & 17 & 130 & 1 & 1.07 \mathrm{E}-35 \\ \text { BP } & 1139 & 48 & 161 & 0.9999512 & 6.45 \mathrm{E}-34 \\ \text { BP } & 890 & 19 & 135 & 1 & 2.64 \mathrm{E}-31 \\ \text { BP } & 601 & 10 & 104 & 1 & 1.02 \mathrm{E}-28 \\ \text { BP } & 402 & 9 & 83 & 0.9999927 & 2.24 \mathrm{E}-28 \\ \text { BP } & 254 & 6 & 64 & 0.9996237 & 4.13 \mathrm{E}-27\end{array}$

Biological processes upregulated in the Jejunum in non-IBD

\section{GO ID}

GO:0003008

GO:0099537

GO:0032501

GO:0099536

GO:0048731

GO:0044057

GO:0098916

GO:0007268

GO:0006936

GO:0032502

GO:0007399

GO:0048468

GO:0048856

GO:0003012

GO:0007275

\section{Term}

system process

trans-synaptic signaling

multicellular organismal process

synaptic signaling

system development

regulation of system process

anterograde trans-synaptic signaling

chemical synaptic transmission

muscle contraction

developmental process

nervous system development

cell development

anatomical structure development

muscle system process

multicellular organism development $\begin{array}{llllll}\text { Ont } & \text { N } & \text { Up } & \text { Down } & \text { P.Up } & \text { P.Down }\end{array}$

$\begin{array}{llllll}\text { BP } & 1229 & 204 & 64 & 3.89 \mathrm{E}-36 & 5.12 \mathrm{E}-01\end{array}$

$\begin{array}{llllll}\mathrm{BP} & 538 & 105 & 20 & 8.64 \mathrm{E}-24 & 9.59 \mathrm{E}-01\end{array}$

$\begin{array}{llllll}\mathrm{BP} & 5121 & 490 & 327 & 1.40 \mathrm{E}-23 & 7.31 \mathrm{E}-07\end{array}$

BP $543 \quad 105 \quad 20 \quad 1.85 \mathrm{E}-23 \quad 9.63 \mathrm{E}-01$

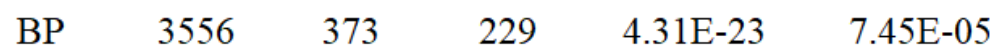

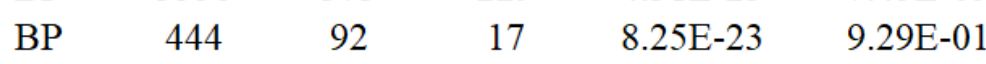

$\begin{array}{llllll}\mathrm{BP} & 530 & 102 & 20 & 1.23 \mathrm{E}-22 & 9.51 \mathrm{E}-01\end{array}$

BP $530 \quad 102 \quad 20 \quad 1.23 \mathrm{E}-22 \quad 9.51 \mathrm{E}-01$

$\begin{array}{llllll}\mathrm{BP} & 267 & 66 & 10 & 7.02 \mathrm{E}-21 & 8.93 \mathrm{E}-01\end{array}$

BP $4618 \quad 444 \quad 281 \quad 1.20 \mathrm{E}-20 \quad 4.77 \mathrm{E}-04$

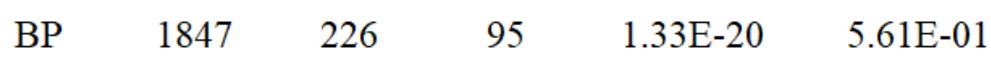

$\begin{array}{llllll}\mathrm{BP} & 1674 & 210 & 98 & 2.52 \mathrm{E}-20 & 1.09 \mathrm{E}-01\end{array}$

$\begin{array}{lllll}\mathrm{BP} & 4314 & 418 & 263 & 1.43 \mathrm{E}-19\end{array}$

$\begin{array}{llllll}\mathrm{BP} & 340 & 73 & 11 & 3.50 \mathrm{E}-19 & 9.69 \mathrm{E}-01\end{array}$

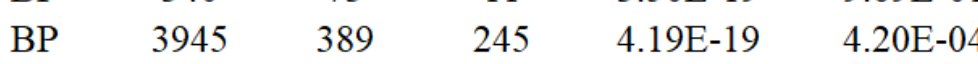

